\title{
Synthetic Control of Excited States in Cyclometalated Ir(III) Complexes using Ancillary Ligands
}

Jian Li, ${ }^{(a)}$ Peter I. Djurovich, ${ }^{(a)}$ Bert D. Alleyne, ${ }^{(a)}$ Muhammed Yousufuddin, ${ }^{(a)}$ Nam N. Ho, ${ }^{(a)}$ J. Christopher Thomas, ${ }^{(b)}$ Jonas C. Peters, $^{(b)}$ Robert Bau, ${ }^{(a)}$ and Mark E. Thompson ${ }^{(a) *}$

(a) University of Southern California, Department of Chemistry, Los Angeles, CA 90089

(b) Division of Chemistry and Chemical Engineering, Arnold and Mabel Beckman Laboratories of Chemical Synthesis, California Institute of Technology, Pasadena, CA 91125

Supporting information.

Figure Captions.

Figure S 1. Abbreviations of Iridium complexes used through out the paper.

Figure S 2. Absorption spectra of $(t p y)_{2} \operatorname{Ir}(\mathrm{pz})_{2} \mathrm{Bpz}_{2}$ in hexane, $\mathrm{CH}_{2} \mathrm{Cl}_{2}, \mathrm{DMSO} .{ }^{1} \mathrm{MLCT}$ absorption spectra are demonstrated in the inset.

Figure $\mathrm{S} 3$. Absorption, emission $(77 \mathrm{~K})$ and excitation $(77 \mathrm{~K})$ spectra of $\left[(t p y)_{2} \operatorname{Ir}(\mathrm{CN}-t-\mathrm{Bu})_{2}\right]\left(\mathrm{CF}_{3} \mathrm{SO}_{3}\right)$.

Figure S 4 Rigidochromic shift vs emission energy of $(t p y)_{2} \operatorname{Ir}(\mathrm{LX})$ complexes in 2-MeTHF. The ancillary ligands (LX) are numbered as in Figure 1.

Figure S 5. Absorption spectra of $(d f p p y)_{2} \operatorname{Ir}\left[\left(\mathrm{PPh}_{2} \mathrm{CH}_{2}\right)_{2} \mathrm{BPh}_{2}\right],(d f p p y)_{2} \operatorname{Ir}\left(\mathrm{pz}_{2} \mathrm{Bpz}_{2}\right)$, and $(d f p p y)_{2} \operatorname{Ir}(\mathrm{acac})$ complexes in $\mathrm{CH}_{2} \mathrm{Cl}_{2}$.

Figure S 6. RT emission spectra of $(d f p p y)_{2} \operatorname{Ir}\left(\mathrm{PPh}_{2} \mathrm{CH}_{2}\right)_{2} \mathrm{BPh}_{2},(d f p p y)_{2} \operatorname{Ir}(\mathrm{pz})_{2} \mathrm{Bpz}_{2},(d f p p y)_{2} \operatorname{Ir}(\mathrm{pz})_{2} \mathrm{H}$, and $(\text { dfppy })_{2} \operatorname{Ir}(\mathrm{acac})$ complexes in 2-MeTHF.

Figure S 7. $77 \mathrm{~K}$ emission spectra of $(d f p p y)_{2} \operatorname{Ir}\left(\mathrm{PPh}_{2} \mathrm{CH}_{2}\right)_{2} \mathrm{BPh}_{2},(d f p p y)_{2} \operatorname{Ir}(\mathrm{pz})_{2} \mathrm{Bpz}_{2},(d f p p y)_{2} \operatorname{Ir}(\mathrm{pz})_{2} \mathrm{H}$, and $(\text { dfppy })_{2} \operatorname{Ir}(\mathrm{acac})$ complexes in 2-MeTHF.

Figure S 8. ORTEP drawings of $(t p y)_{2} \mathrm{Ir}\left(\mathrm{PPh}_{2} \mathrm{CH}_{2}\right)_{2} \mathrm{BPh}_{2} \cdot \mathrm{H}_{2} \mathrm{O}$ The thermal ellipsoids for the image represent $25 \%$ probability limit. The hydrogen atoms, counter anion and solvent are omitted for clarity.

Figure S 9. ORTEP drawings of $(t p y)_{2} \operatorname{Ir}(\mathrm{CN}-t-\mathrm{Bu})_{2}\left(\mathrm{CF}_{3} \mathrm{SO}_{3}\right) \cdot \mathrm{CHCl}_{3}$. The thermal ellipsoids for the image represent $25 \%$ probability limit. The hydrogen atoms, counter anion and solvent are omitted for clarity. 
Table Captions.

Table S 1. Selected parameters for plot of $k_{\mathrm{r}}$ vs. $\frac{1}{\Delta E^{2}}\left(\frac{v_{\mathrm{T}_{1}}}{v_{{ }_{1} M L C T}}\right)^{3}$ and plot of $\varepsilon$ vs. $\frac{1}{\Delta E^{2}} \frac{v_{\mathrm{T}_{1}}}{v_{{ }{ }_{M L C T}}}$ of $(t p y)_{2} \operatorname{Ir}(\mathrm{LL}$ ') complexes.

Table S 2. Redox properties of $(d f p p y)_{2} \mathrm{Ir}\left(\mathrm{LL}^{\prime}\right)$ complexes. Redox measurements were carried out in DMF solution unless noted: (a) in $\mathrm{CH}_{2} \mathrm{Cl}_{2}$; (b) in $\mathrm{CH}_{3} \mathrm{CN}$. The redox values are reported relative to $\mathrm{Fc}^{+} / \mathrm{Fc}$. The electrochemical process is reversible unless noted:(c) Quasi-reversible; (d) Irreversible.

Table S 3. Photophysical properties of $(d f p p y)_{2} \operatorname{Ir}(\mathrm{LX})$ complexes. Absorption measurements were carried out in $\mathrm{CH}_{2} \mathrm{Cl}_{2}$. Emission and lifetime measurements were carried out in 2-methyltetrahydrofuran (2MeTHF).

Table S 4. Selected excited state properties and IR absorption of selected ( $d f p p y)_{2} \operatorname{Ir}(\mathrm{LX})$ complexes.

Table S 5. Atomic coordinates $\left(\times 10^{4}\right)$ and equivalent isotropic displacement parameters $\left(\AA^{2} \times 10^{3}\right)$ for $(\text { tpy })_{2} \operatorname{Ir}\left(\mathrm{PPh}_{2} \mathrm{CH}_{2}\right)_{2} \mathrm{BPh}_{2} \cdot \mathrm{H}_{2} \mathrm{O}$. U(eq) is defined as one third of the trace of the orthogonalized $\mathrm{U}^{\mathrm{ij}}$ tensor.

Table S 6. Bond lengths $[\AA]$ for $(t p y)_{2} \operatorname{Ir}\left(\mathrm{PPh}_{2} \mathrm{CH}_{2}\right)_{2} \mathrm{BPh}_{2} \cdot \mathrm{H}_{2} \mathrm{O}$.

Table S 7. Bond angles $\left[^{\circ}\right]$ for $(t p y)_{2} \operatorname{Ir}\left(\mathrm{PPh}_{2} \mathrm{CH}_{2}\right)_{2} \mathrm{BPh}_{2} \cdot \mathrm{H}_{2} \mathrm{O}$.

Table S 8. Anisotropic displacement parameters $\left(\AA^{2} \times 10^{3}\right)$ for $(t p y)_{2} \operatorname{Ir}\left(\mathrm{PPh}_{2} \mathrm{CH}_{2}\right)_{2} \mathrm{BPh}_{2} \cdot \mathrm{H}_{2} \mathrm{O}$.. The anisotropic displacement factor exponent takes the form: $-2 \pi^{2}\left[\mathrm{~h}^{2} \mathrm{a}^{2} \mathrm{U}^{11}+\ldots+2 \mathrm{hka} * \mathrm{~b} * \mathrm{U}^{12}\right]$

Table S 9. Atomic coordinates ( $\left.\mathrm{x} 10^{4}\right)$ and equivalent isotropic displacement parameters $\left(\AA^{2} \times 10^{3}\right)$ for $\left[(t p y)_{2} \mathrm{Ir}(\mathrm{CN}-t-\mathrm{Bu})_{2}\right]\left(\mathrm{CF}_{3} \mathrm{SO}_{3}\right) \cdot \mathrm{CHCl}_{3}$. U(eq) is defined as one third of the trace of the orthogonalized $\mathrm{U}^{\mathrm{ij}}$ tensor.

Table S 10. Bond lengths $[\AA]$ and angles $\left[^{\circ}\right]$ for $\left[(t p y)_{2} \operatorname{Ir}(\mathrm{CN}-t-\mathrm{Bu})_{2}\right]\left(\mathrm{CF}_{3} \mathrm{SO}_{3}\right) \cdot \mathrm{CHCl}_{3}$.

Table S 11. Bond angles [ $\left.{ }^{\circ}\right]$ for $\left[(t p y)_{2} \mathrm{Ir}(\mathrm{CN}-t-\mathrm{Bu})_{2}\right]\left(\mathrm{CF}_{3} \mathrm{SO}_{3}\right) \cdot \mathrm{CHCl}_{3}$.

Table S 12. Anisotropic displacement parameters $\left(\AA^{2} \times 10^{3}\right)$ for $\left[(t p y)_{2} \mathrm{Ir}(\mathrm{CN}-t-\mathrm{Bu})_{2}\right]\left(\mathrm{CF}_{3} \mathrm{SO}_{3}\right) \cdot \mathrm{CHCl}_{3}$. The anisotropic displacement factor exponent takes the form: $-2 \pi^{2}\left[\mathrm{~h}^{2} \mathrm{a}^{*} \mathrm{U}^{11}+\ldots+2 \mathrm{hka} \mathrm{b}^{*} \mathrm{U}^{12}\right]$.

Table S 13. Hydrogen coordinates $\left(\times 10^{4}\right)$ and isotropic displacement parameters $\left(\AA^{2} \times 10^{3}\right)$ for $\left[(t p y)_{2} \mathrm{Ir}(\mathrm{CN}-t-\mathrm{Bu})_{2}\right]\left(\mathrm{CF}_{3} \mathrm{SO}_{3}\right) \cdot \mathrm{CHCl}_{3}$. 
Figure S 1. Abbreviations of Iridium complexes used through out the paper.
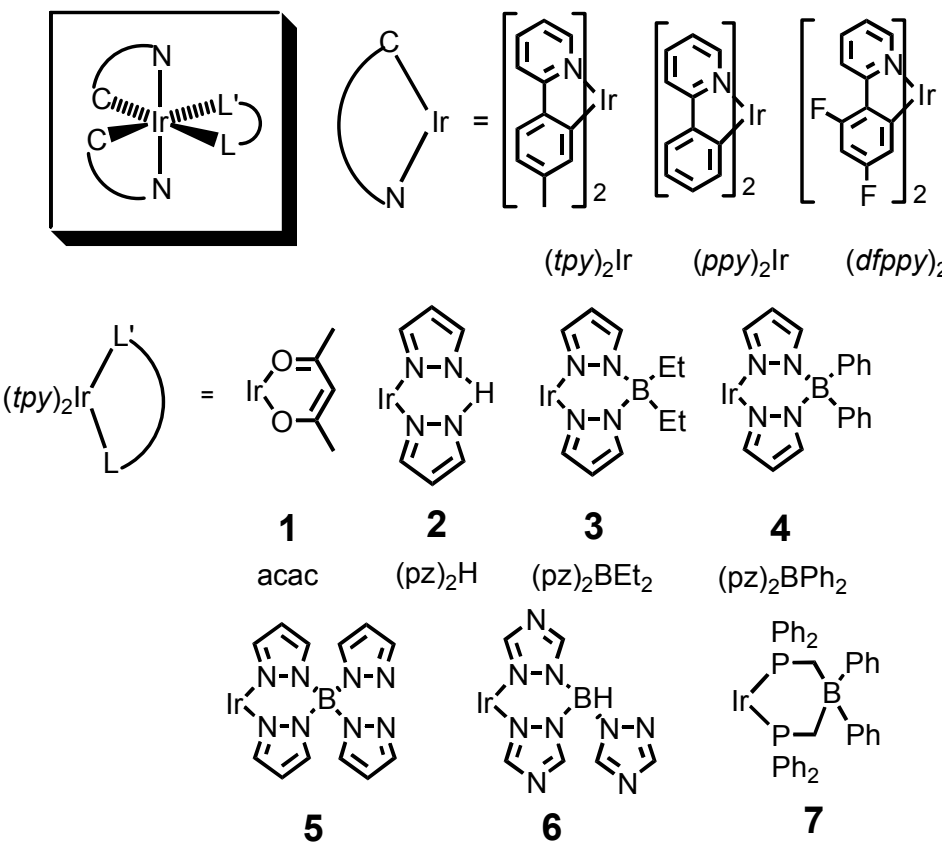

$$
(\mathrm{pz})_{2} \mathrm{Bpz}_{2} \quad(\mathrm{tz})_{3} \mathrm{BH} \quad\left(\mathrm{PPh}_{2} \mathrm{CH}_{2}\right)_{2} \mathrm{BPh}_{2}
$$

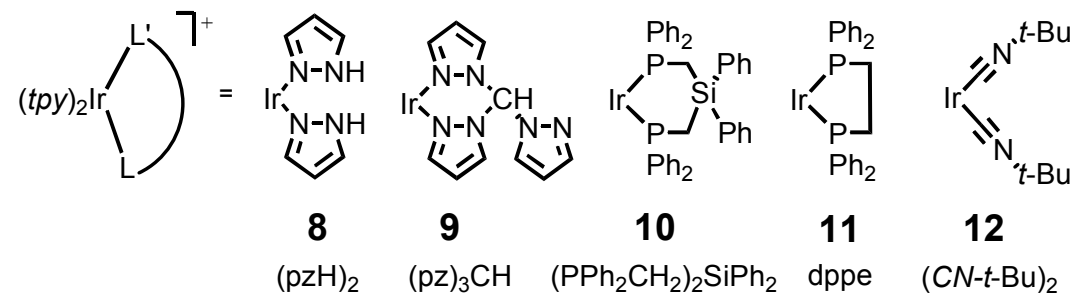

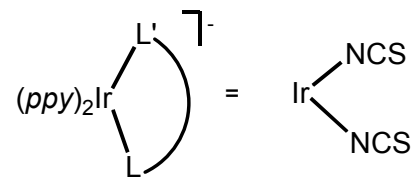

13

$(\mathrm{NCS})_{2}$<smiles>N#C[I-]</smiles>

14

$(\mathrm{CN})_{2}$ 
Figure S 2. Absorption spectra of (tpy)2Ir(pz)2Bpz2 in hexane, CH2C12, DMSO. ${ }^{\text {MLCT }}$ absorption spectra are demonstrated in the inset.

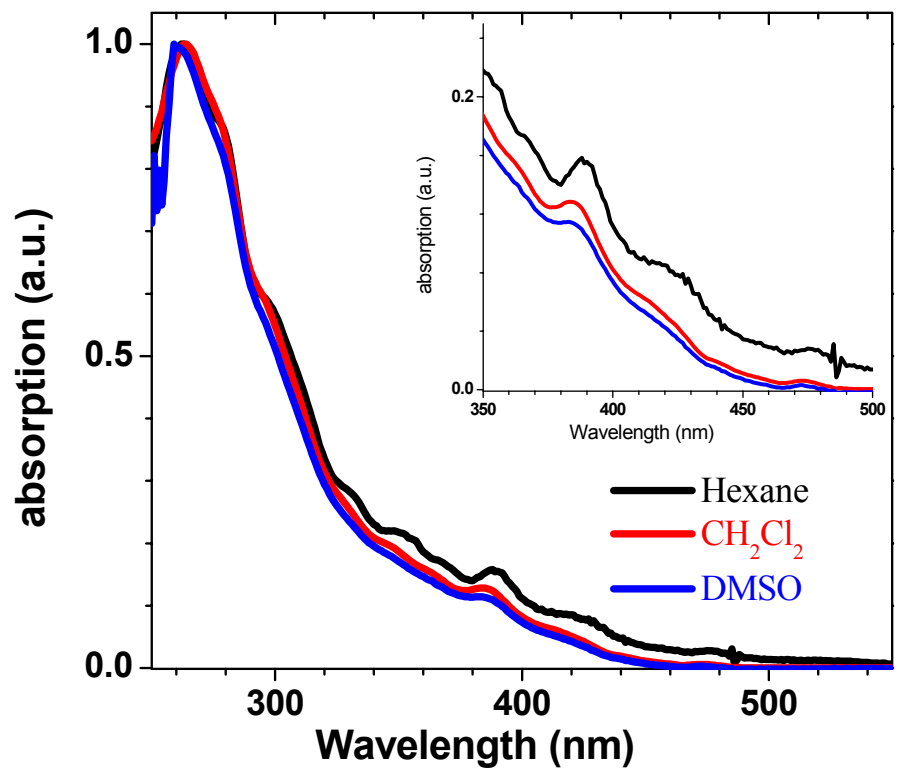

Figure S 3. Absorption, emission (77 K) and excitation (77 K) spectra of [(tpy)2Ir(CN-t-Bu)2](CF3SO3).

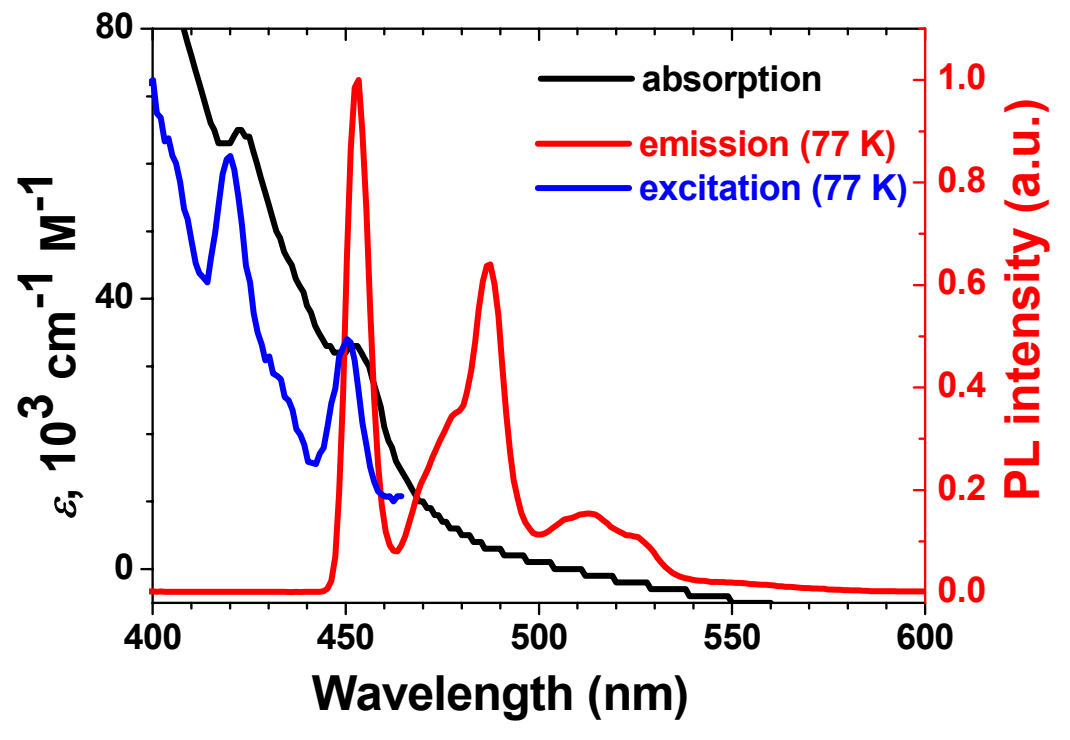


Figure S 4 Rigidochromic shift vs emission energy of $(t p y)_{2} \operatorname{Ir}(\mathrm{LX})$ complexes in 2-MeTHF. The ancillary ligands (LX) are numbered as in Figure 1.

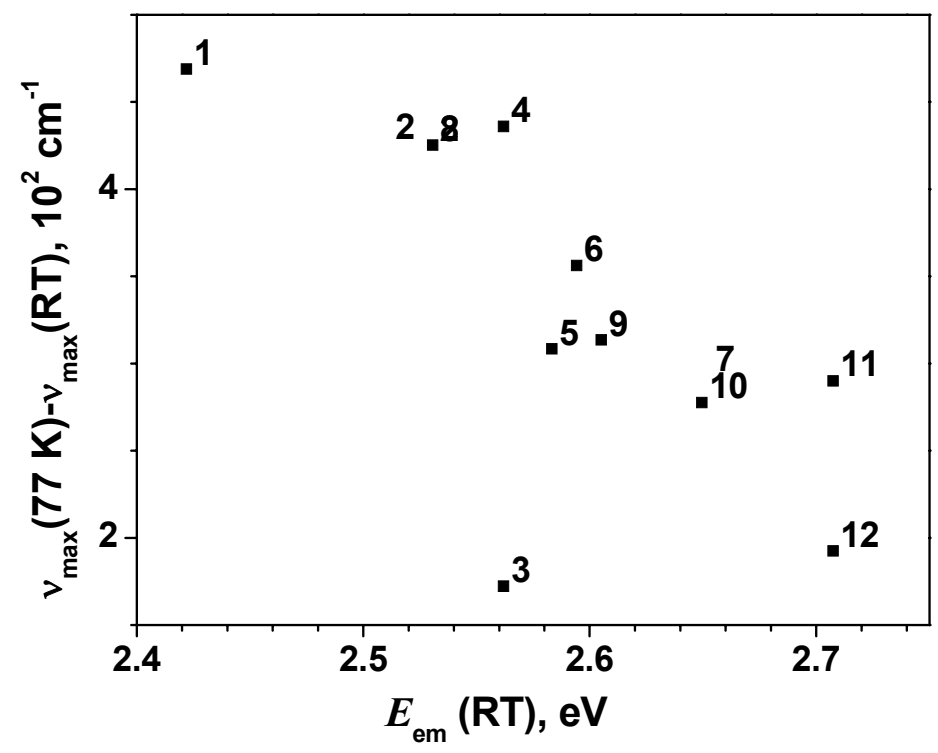

Figure S 5. Absorption spectra of (dfppy)2Ir[( $\mathrm{PPh} 2 \mathrm{CH} 2) 2 \mathrm{BPh} 2]$, (dfppy)2Ir(pz2Bpz2), and (dfppy)2Ir(acac) complexes in $\mathrm{CH} 2 \mathrm{Cl} 2$.

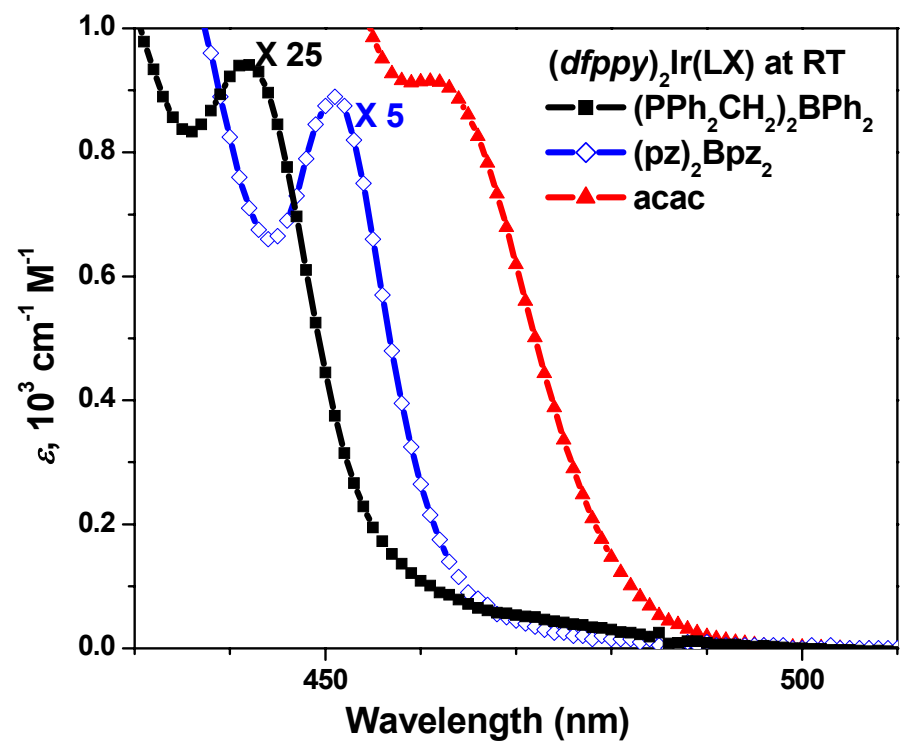


Figure S 6. RT emission spectra of (dfppy)2 $\operatorname{Ir}(\mathrm{PPh} 2 \mathrm{CH} 2) 2 \mathrm{BPh} 2$, (dfppy)2 $\operatorname{Ir}(\mathrm{pz}) 2 \mathrm{Bpz} 2$, (dfppy)2Ir(pz)2H, and (dfppy)2Ir(acac) complexes in 2-MeTHF.

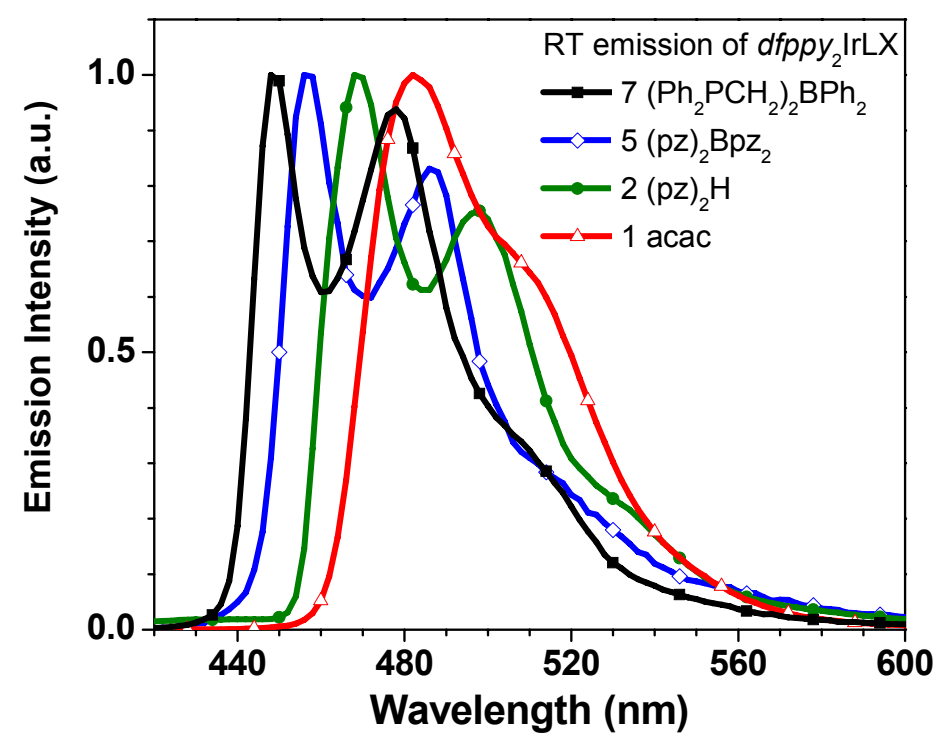

Figure S 7. $77 \mathrm{~K}$ emission spectra of (dfppy)2 $\operatorname{Ir}(\mathrm{PPh} 2 \mathrm{CH} 2) 2 \mathrm{BPh} 2$, (dfppy)2 $\operatorname{Ir}(\mathrm{pz}) 2 \mathrm{Bpz} 2$, (dfppy)2 $\operatorname{Ir}(\mathrm{pz}) 2 \mathrm{H}$, and (dfppy)2Ir(acac) complexes in 2-MeTHF.

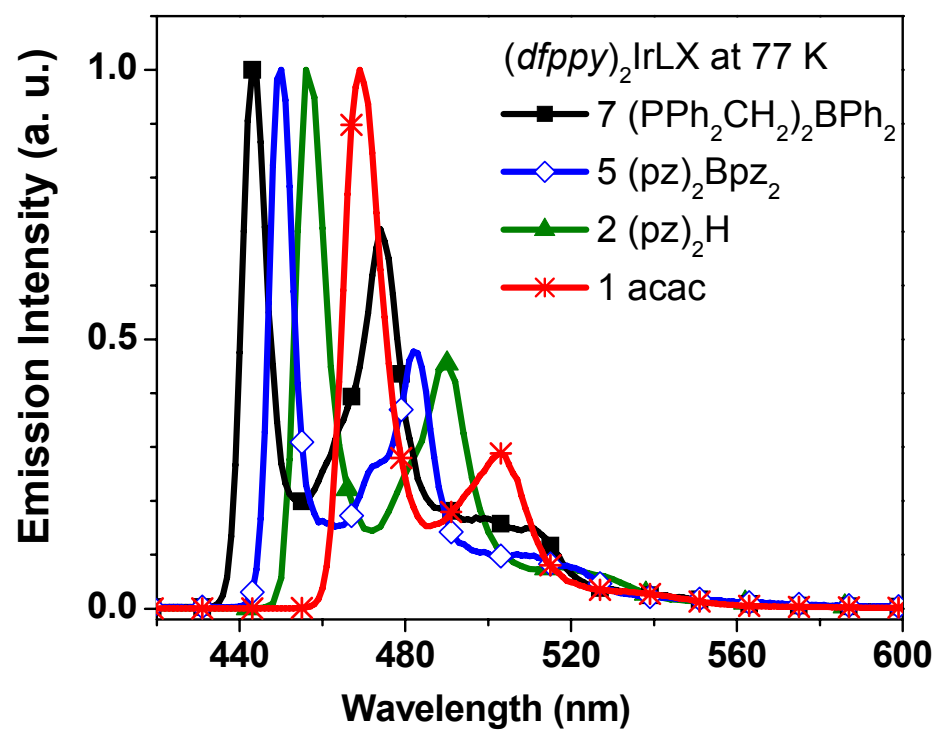


Figure S 8. ORTEP drawings of $(t p y)_{2} \operatorname{Ir}\left(\mathrm{PPh}_{2} \mathrm{CH}_{2}\right)_{2} \mathrm{BPh}_{2} \cdot \mathrm{H}_{2} \mathrm{O}$ The thermal ellipsoids for the image represent $25 \%$ probability limit. The hydrogen atoms, counter anion and solvent are omitted for clarity.

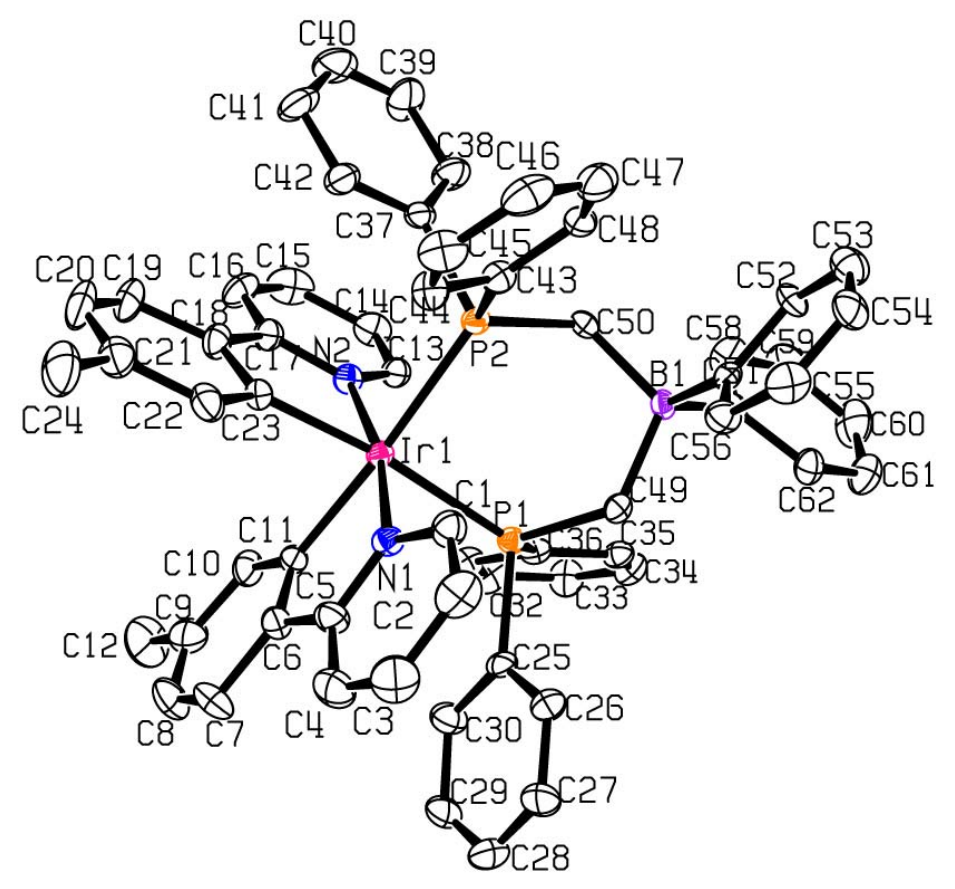

Figure S 9. ORTEP drawings of (tpy) $2 \operatorname{Ir}(\mathrm{CN}-\mathrm{t}-\mathrm{Bu}) 2(\mathrm{CF} 3 \mathrm{SO} 3) \cdot \mathrm{CHCl}$. The thermal ellipsoids for the image represent $25 \%$ probability limit. The hydrogen atoms, counter anion and solvent are omitted for clarity.

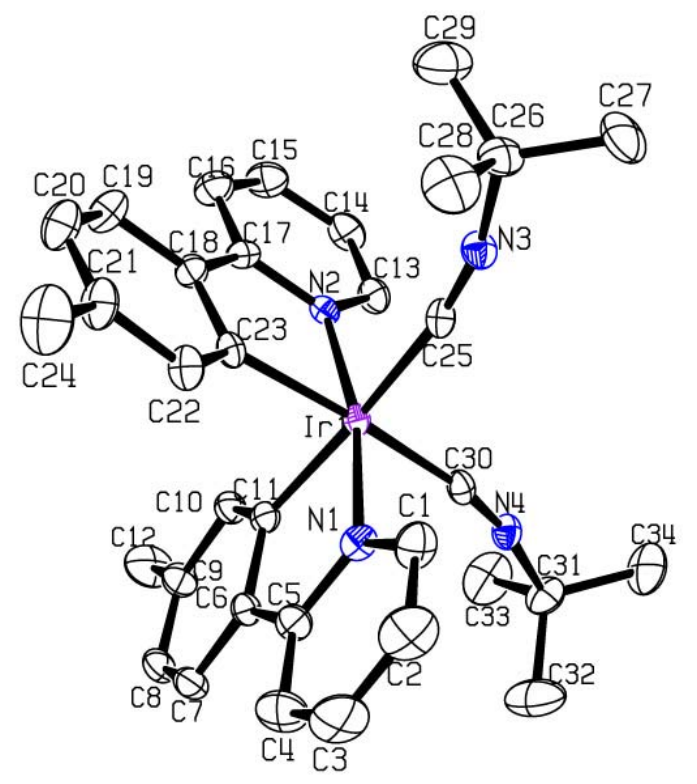


Table S 1. Selected parameters for plot of $k_{\mathrm{r}}$ vs. $\frac{1}{\Delta E^{2}}\left(\frac{v_{\mathrm{T}_{1}}}{v_{{ }^{1} M L C T}}\right)^{3}$ and plot of $\varepsilon$ vs. $\frac{1}{\Delta E^{2}} \frac{v_{\mathrm{T}_{1}}}{v_{1^{1} M L C T}}$ of $(t p y)_{2} \operatorname{Ir}(\mathrm{LL}$ ') complexes.

\begin{tabular}{|c|c|c|c|c|c|c|c|}
\hline$\left(C^{\wedge} N\right)_{2} \operatorname{Ir}\left(\mathrm{LL}^{\prime}\right)$ & $\begin{array}{l}U_{{ }_{1} M L C T}, \\
10^{4} \mathrm{~cm}^{-1}\end{array}$ & $\begin{array}{c}\Delta \mathrm{E} \\
10^{3} \mathrm{~cm}^{-1}\end{array}$ & $\begin{array}{c}v_{T_{1}} \\
10^{4} \mathrm{~cm}^{-1}\end{array}$ & $\begin{array}{c}\left|\frac{1}{\Delta \mathrm{E}}\right|^{2} \frac{v_{T_{1}}}{v_{{ }_{M L C T}}{ }^{3}} \\
10^{-16} \mathrm{~cm}^{-4}\end{array}$ & $\begin{array}{c}\varepsilon, \\
10^{3} \mathrm{~cm}^{-1} \mathrm{M}^{-1}\end{array}$ & $\begin{array}{c}\left|\frac{1}{\Delta E}\right|^{2}\left(\frac{v_{T 1}}{v_{{ }_{1}}}\right) \\
10^{-7} \mathrm{~cm}^{-2}\end{array}$ & $\begin{array}{l}k_{\mathrm{r}} \\
10^{5} \mathrm{~s}^{-1}\end{array}$ \\
\hline $\mathbf{1}(\text { tpy })_{2} \operatorname{Ir}(\mathrm{acac})$ & 2.46 & 2.1 & 2.05 & 3.09 & 1.0 & 1.30 & 2.7 \\
\hline $2(t p y)_{2} \operatorname{Ir}(\mathrm{pz})_{2} \mathrm{H}$ & $2 . .55$ & 2.99 & 2.09 & 1.41 & 0.42 & 0.618 & 2.0 \\
\hline $\mathbf{3}(t p y)_{2} \operatorname{Ir}(\mathrm{pz})_{2} \mathrm{BEt}_{2}$ & 2.56 & 3.12 & 2.10 & 1.28 & 0.35 & 0.562 & 2.0 \\
\hline $4(t p y)_{2} \operatorname{Ir}(\mathrm{pz})_{2} \mathrm{BPh}_{2}$ & 2.56 & 3.05 & 2.11 & 1.35 & 0.31 & 0.599 & 1.4 \\
\hline $5(t p y)_{2} \operatorname{Ir}(\mathrm{pz})_{2} \mathrm{Bpz}_{2}$ & 2.61 & 3.59 & 2.12 & 0.93 & 0.25 & 0.415 & 1.5 \\
\hline $\mathbf{6}(t p y)_{2} \operatorname{Ir}(\mathrm{tz})_{3} \mathrm{BH}$ & 2.62 & 3.66 & 2.12 & 0.89 & 0.16 & 0.399 & 1.3 \\
\hline $7(t p y)_{2} \mathrm{Ir}\left(\mathrm{PPh}_{2} \mathrm{CH}_{2}\right)_{2} \mathrm{BPh}_{2}$ & 2.70 & 4.50 & 2.16 & 0.54 & 0.04 & 0.252 & 0.08 \\
\hline $8\left[(t p y)_{2} \operatorname{Ir}(\mathrm{pzH})_{2}\right]\left(\mathrm{CF}_{3} \mathrm{SO}_{3}\right)$ & 2.62 & 3.65 & 2.12 & 0.88 & 0.17 & 0.397 & 1.1 \\
\hline $\mathbf{9}\left[(t p y)_{2} \mathrm{Ir}(\mathrm{pz})_{3} \mathrm{CH}\right]\left(\mathrm{CF}_{3} \mathrm{SO}_{3}\right)$ & 2.65 & 4.00 & 2.13 & 0.71 & 0.16 & 0.324 & 1.1 \\
\hline $\begin{array}{l}\mathbf{1 0}\left[(t p y)_{2} \mathrm{Ir}\left(\mathrm{PPh}_{2} \mathrm{CH}_{2}\right)_{2} \mathrm{SiPh}_{2}\right] \\
\left(\mathrm{CF}_{3} \mathrm{SO}_{3}\right)\end{array}$ & 2.78 & 5.26 & 2.16 & 0.36 & 0.04 & 0.169 & 0.05 \\
\hline $11\left[(t p y)_{2} \operatorname{Ir}(\mathrm{dppe})\right]\left(\mathrm{CF}_{3} \mathrm{SO}_{3}\right)$ & 2.79 & 5.41 & 2.19 & 0.34 & 0.03 & 0.165 & 0.03 \\
\hline $\begin{array}{l}12\left[(t p y)_{2} \operatorname{Ir}(\mathrm{CN}-t-\mathrm{Bu})_{2}\right] \\
\left(\mathrm{CF}_{3} \mathrm{SO}_{3}\right)\end{array}$ & 2.87 & 6.21 & 2.21 & 0.24 & 0.03 & 0.118 & 0.08 \\
\hline $13\left(\mathrm{NBu}_{4}\right)\left[(\text { ppy })_{2} \operatorname{Ir}(\mathrm{NCS})_{2}\right]$ & 2.51 & 2.60 & 2.07 & 1.93 & 0.59 & 0.831 & 2.7 \\
\hline $14\left(\mathrm{NBu}_{4}\right)\left[(p p y)_{2} \operatorname{Ir}(\mathrm{CN})_{2}\right]$ & 2.62 & 3.72 & 2.16 & 0.86 & 0.25 & 0.404 & 2.0 \\
\hline
\end{tabular}


Table S 2. Redox properties of (dfppy)2Ir(LL') complexes. Redox measurements were carried out in DMF solution unless noted: (a) in $\mathrm{CH} 2 \mathrm{Cl} 2$; (b) in $\mathrm{CH} 3 \mathrm{CN}$. The redox values are reported relative to $\mathrm{Fc}+/ \mathrm{Fc}$. The electrochemical process is reversible unless noted:(c) Quasi-reversible; (d) Irreversible.

\begin{tabular}{lccc}
\hline \multicolumn{1}{c}{$\left(C^{\wedge} N\right)_{2} \operatorname{Ir}(\mathrm{LX})$} & $\mathrm{E}_{1 / 2}^{\mathrm{Ox}}(\mathrm{V})$ & $\mathrm{E}_{1 / 2}^{\mathrm{Red}}(\mathrm{V})$ & $\Delta \mathrm{E}(\mathrm{V})$ \\
\hline $1(\text { dfppy })_{2} \operatorname{Ir}(\mathrm{acac})$ & 0.76 & -2.48 & 3.24 \\
$2(\text { dfppy })_{2} \operatorname{Ir}(\mathrm{pz})_{2} \mathrm{H}$ & $0.63^{\mathrm{a}, \mathrm{d}}, 0.93^{\mathrm{a}, \mathrm{d}}$ & $-2.44^{\mathrm{c}}$ & $3.07,3.37$ \\
$5(\text { dfppy })_{2} \operatorname{Ir}(\mathrm{pz})_{2} \mathrm{Bpz}_{2}$ & $1.08^{\mathrm{a}, \mathrm{c}}$ & -2.39 & 3.47 \\
$7(\text { dfppy })_{2} \operatorname{Ir}\left(\mathrm{PPh}_{2} \mathrm{CH}_{2}\right)_{2} \mathrm{BPh}_{2}$ & $0.95^{\mathrm{a}, \mathrm{d}}$ & -2.38 & 3.33 \\
$12\left[(\text { dfppy })_{2} \operatorname{Ir}(\mathrm{CN}-t-\mathrm{Bu})_{2}\right]\left(\mathrm{CF}_{3} \mathrm{SO}_{3}\right)$ & $>1.40^{\mathrm{b}}$ & $-2.19^{\mathrm{d}}$ & $>3.59$ \\
\hline
\end{tabular}

Table S 3. Photophysical properties of (dfppy) $2 \operatorname{Ir}(\mathrm{LX})$ complexes. Absorption measurements were carried out in $\mathrm{CH} 2 \mathrm{Cl} 2$. Emission and lifetime measurements were carried out in 2-methyltetrahydrofuran (2MeTHF).

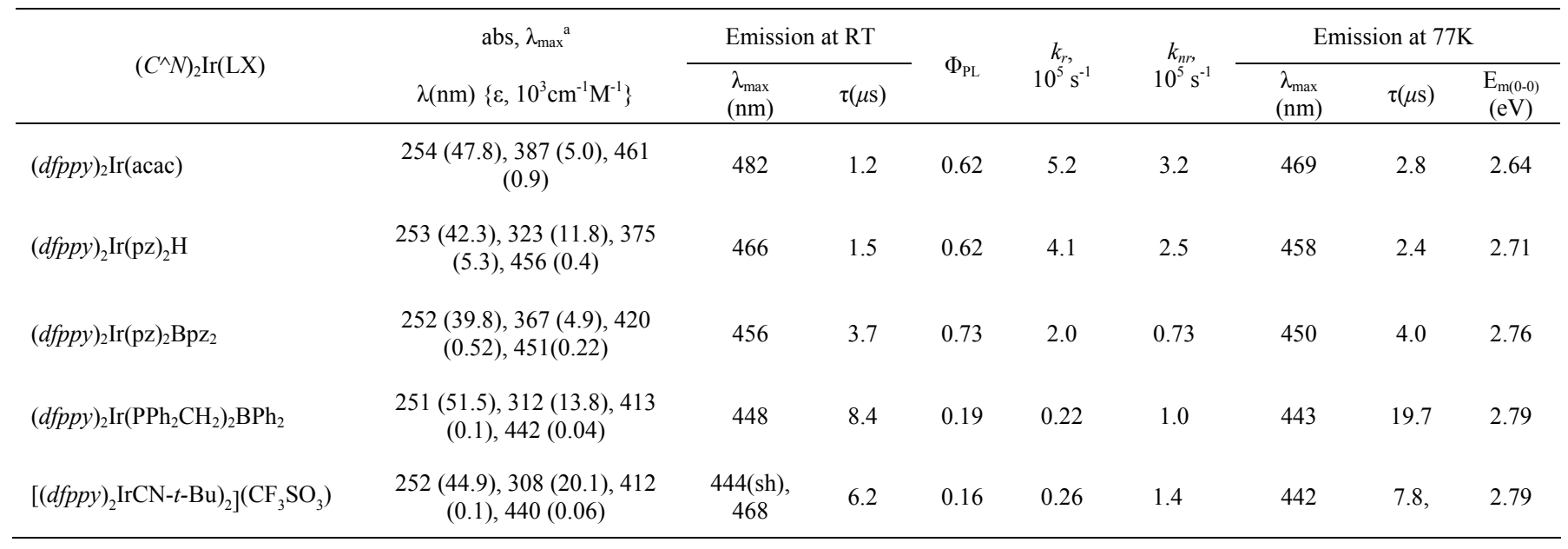

Table S 4. Selected excited state properties and IR absorption of selected ( $d f p p y)_{2} \operatorname{Ir}(\mathrm{LX})$ complexes.

\begin{tabular}{|c|c|c|c|c|}
\hline$(d f p p y)_{2} \operatorname{Ir}(\mathrm{LX})$ & $\begin{array}{c}E_{\mathrm{em}}(0-0) \\
\left(\mathrm{x} 10^{6} \mathrm{~cm}^{-1}\right)\end{array}$ & $\hbar \omega_{M}\left(\mathrm{~cm}^{-1}\right)$ & $\mathrm{S}_{\mathrm{M}}$ & $\begin{array}{c}\text { IR absorption } \\
\left(1400-1550 \mathrm{~cm}^{-1}\right)\end{array}$ \\
\hline$(d f \mathrm{p} p y)_{2} \operatorname{Ir}(\mathrm{acac})$ & 2.13 & 1441 & 0.29 & $\begin{array}{c}1515,1478,1428 \\
1403\end{array}$ \\
\hline$(d f p p y)_{2} \operatorname{Ir}(\mathrm{pz})_{2} \mathrm{H}$ & 2.18 & 1426 & 0.46 & $1478,1428,1404$ \\
\hline$(d f p p y)_{2} \operatorname{Ir}(\mathrm{pz})_{2} \mathrm{Bpz}_{2}$ & 2.22 & 1475 & 0.48 & $\begin{array}{c}1507,1478,1430 \\
1404\end{array}$ \\
\hline$(d f p p y)_{2} \operatorname{Ir}\left(\mathrm{PPh}_{2} \mathrm{CH}_{2}\right)_{2} \mathrm{BPh}_{2}$ & 2.26 & 1475 & 0.70 & $1479,1434,1406$ \\
\hline$\left[(d f p p y)_{2} \operatorname{Ir}(\mathrm{CN}-t-\mathrm{Bu})_{2}\right]\left(\mathrm{CF}_{3} \mathrm{SO}_{3}\right)$ & 2.26 & 1437 & 0.87 & $1480,1431,1408$ \\
\hline free $d f p p y$ ligand & 2.36 & 1468 & 2.22 & $\begin{array}{c}1505,1466,1440 \\
1416\end{array}$ \\
\hline
\end{tabular}


Table S 5. Atomic coordinates (x104) and equivalent isotropic displacement parameters ( $\AA 2 \times 103)$ for (tpy) $2 \operatorname{Ir}(\mathrm{PPh} 2 \mathrm{CH} 2) 2 \mathrm{BPh} 2 \cdot \mathrm{H} 2 \mathrm{O}$. U(eq) is defined as one third of the trace of the orthogonalized Uij tensor.

\begin{tabular}{|c|c|c|c|c|}
\hline & $\mathrm{x}$ & $\mathrm{y}$ & $\mathrm{z}$ & $\mathrm{U}(\mathrm{eq})$ \\
\hline $\operatorname{Ir}(1)$ & $9848(1)$ & $2704(1)$ & 1783(1) & $31(1)$ \\
\hline $\mathrm{N}(1)$ & $8858(4)$ & $3499(5)$ & $1828(3)$ & $36(2)$ \\
\hline $\mathrm{N}(2)$ & $10800(4)$ & 2011(6) & 1581(3) & $38(2)$ \\
\hline $\mathrm{B}(1)$ & $10164(6)$ & 1691(7) & $3483(4)$ & $33(2)$ \\
\hline $\mathrm{P}(1)$ & $9243(1)$ & $1434(2)$ & $2232(1)$ & $32(1)$ \\
\hline $\mathrm{P}(2)$ & $10785(1)$ & $3052(2)$ & 2693(1) & $33(1)$ \\
\hline$C(1)$ & $8755(6)$ & $3944(8)$ & $2305(5)$ & $49(2)$ \\
\hline$C(2)$ & $8077(7)$ & $4429(9)$ & $2329(6)$ & $68(3)$ \\
\hline$C(3)$ & $7468(8)$ & $4449(11)$ & 1821(6) & $83(4)$ \\
\hline$C(4)$ & 7572(7) & $3997(10)$ & $1346(6)$ & $70(3)$ \\
\hline$C(5)$ & $8279(6)$ & $3500(8)$ & $1349(5)$ & $49(3)$ \\
\hline$C(6)$ & $8441(6)$ & $2955(8)$ & $877(4)$ & $50(3)$ \\
\hline$C(7)$ & $7873(8)$ & $2886(12)$ & $319(6)$ & $83(5)$ \\
\hline $\mathrm{C}(8)$ & $8075(10)$ & $2321(11)$ & $-94(6)$ & $87(5)$ \\
\hline$C(9)$ & $8749(8)$ & $1779(11)$ & $7(6)$ & $70(3)$ \\
\hline $\mathrm{C}(10)$ & $9264(7)$ & $1849(8)$ & $538(4)$ & $54(3)$ \\
\hline $\mathrm{C}(11)$ & $9118(6)$ & $2433(7)$ & $984(4)$ & $41(2)$ \\
\hline $\mathrm{C}(12)$ & $8923(11)$ & $1106(13)$ & $-460(5)$ & $108(6)$ \\
\hline $\mathrm{C}(13)$ & $11062(6)$ & 1127(7) & $1752(4)$ & $43(2)$ \\
\hline$C(14)$ & $11671(6)$ & $672(9)$ & $1595(5)$ & $57(3)$ \\
\hline$C(15)$ & $12057(7)$ & $1230(10)$ & $1224(6)$ & $74(4)$ \\
\hline$C(16)$ & 11793(7) & $2110(9)$ & $1059(5)$ & $60(3)$ \\
\hline$C(17)$ & $11177(6)$ & $2536(7)$ & $1245(4)$ & $43(2)$ \\
\hline$C(18)$ & $10879(6)$ & $3508(8)$ & 1096(4) & $47(2)$ \\
\hline$C(19)$ & 11171(8) & 4182(9) & $752(5)$ & $63(3)$ \\
\hline$C(20)$ & 10893(9) & $5085(10)$ & $648(5)$ & $75(4)$ \\
\hline$C(21)$ & 10263(8) & $5397(9)$ & $864(5)$ & $65(3)$ \\
\hline$C(22)$ & $9945(7)$ & $4762(8)$ & $1207(4)$ & $53(3)$ \\
\hline $\mathrm{C}(23)$ & $10240(6)$ & $3787(7)$ & 1333(4) & $38(2)$ \\
\hline
\end{tabular}




\begin{tabular}{|c|c|c|c|c|}
\hline $\mathrm{C}(24)$ & $9913(10)$ & $6409(10)$ & $748(6)$ & $91(5)$ \\
\hline$C(25)$ & $8167(5)$ & 1394(7) & $1926(4)$ & $38(2)$ \\
\hline$C(26)$ & $7663(6)$ & 1798(8) & $2214(4)$ & $49(2)$ \\
\hline $\mathrm{C}(27)$ & $6875(7)$ & 1761(11) & $1984(5)$ & $73(4)$ \\
\hline $\mathrm{C}(28)$ & $6562(7)$ & 1281(10) & $1477(6)$ & $69(4)$ \\
\hline $\mathrm{C}(29)$ & $7045(7)$ & $887(10)$ & $1190(5)$ & $64(3)$ \\
\hline$C(30)$ & $7861(6)$ & $925(9)$ & $1404(5)$ & $54(3)$ \\
\hline $\mathrm{C}(31)$ & $9537(6)$ & $-226(8)$ & $1612(5)$ & $49(2)$ \\
\hline$C(32)$ & $9644(7)$ & $-1209(8)$ & $1552(5)$ & $60(3)$ \\
\hline$C(33)$ & $9663(7)$ & $-1840(8)$ & $1985(5)$ & $58(3)$ \\
\hline$C(34)$ & $9565(6)$ & $-1503(8)$ & $2495(5)$ & $53(3)$ \\
\hline$C(35)$ & $9472(5)$ & $-528(7)$ & $2584(4)$ & $40(2)$ \\
\hline$C(36)$ & $9454(5)$ & $125(6)$ & $2148(4)$ & $37(2)$ \\
\hline$C(37)$ & $11774(5)$ & $3226(8)$ & $2573(4)$ & $41(2)$ \\
\hline$C(38)$ & $12350(7)$ & $2536(9)$ & $2736(6)$ & $60(3)$ \\
\hline $\mathrm{C}(39)$ & $13088(8)$ & $2658(12)$ & $2612(7)$ & $82(5)$ \\
\hline$C(40)$ & $13216(8)$ & $3521(14)$ & $2360(6)$ & $90(5)$ \\
\hline$C(41)$ & $12649(7)$ & $4189(10)$ & $2186(5)$ & $70(4)$ \\
\hline $\mathrm{C}(42)$ & $11926(6)$ & $4087(8)$ & $2301(4)$ & $52(3)$ \\
\hline$C(43)$ & 10743(6) & 4191(7) & $3095(4)$ & $41(2)$ \\
\hline $\mathrm{C}(44)$ & $10448(6)$ & $5032(8)$ & $2804(5)$ & $53(3)$ \\
\hline$C(45)$ & $10462(7)$ & $5907(8)$ & $3082(6)$ & $62(3)$ \\
\hline$C(46)$ & 10763(8) & 5966(9) & $3668(6)$ & $71(4)$ \\
\hline $\mathrm{C}(47)$ & $11056(8)$ & $5152(10)$ & $3970(5)$ & $67(3)$ \\
\hline $\mathrm{C}(48)$ & $11068(6)$ & $4258(8)$ & $3684(4)$ & $47(2)$ \\
\hline$C(49)$ & $9315(5)$ & $1666(6)$ & 2991(3) & $33(2)$ \\
\hline$C(50)$ & $10902(5)$ & $2040(6)$ & $3204(3)$ & $34(2)$ \\
\hline$C(51)$ & $10094(5)$ & $2430(6)$ & $4016(4)$ & $34(2)$ \\
\hline$C(52)$ & $10679(6)$ & $2366(7)$ & $4542(4)$ & $43(2)$ \\
\hline $\mathrm{C}(53)$ & $10694(8)$ & 2961(8) & 4997(5) & $57(3)$ \\
\hline $\mathrm{C}(54)$ & $10102(7)$ & $3656(8)$ & $4976(4)$ & $56(3)$ \\
\hline$C(55)$ & $9557(7)$ & $3743(8)$ & $4490(5)$ & $58(3)$ \\
\hline$C(56)$ & $9540(6)$ & $3137(8)$ & $3997(4)$ & $45(2)$ \\
\hline$C(57)$ & $10340(6)$ & $613(7)$ & $3777(4)$ & $39(2)$ \\
\hline $\mathrm{C}(58)$ & 10992(7) & $32(8)$ & $3780(5)$ & $63(3)$ \\
\hline $\mathrm{C}(59)$ & 11074(9) & $-882(11)$ & $4038(6)$ & $92(5)$ \\
\hline
\end{tabular}




$\begin{array}{lcccr}\mathrm{C}(60) & 10540(10) & -1256(10) & 4307(6) & 82(4) \\ \mathrm{C}(61) & 9907(8) & -704(8) & 4314(5) & 62(3) \\ \mathrm{C}(62) & 9815(6) & 193(8) & 4069(4) & 49(2) \\ \mathrm{O}(1) & 7390(4) & 2999(10) & 3581(3) & 115(4)\end{array}$

Table S 6. Bond lengths $[\AA]$ for (tpy)2Ir(PPh2CH2)2BPh2 $\cdot \mathrm{H} 2 \mathrm{O}$.

\begin{tabular}{ll}
\hline $\operatorname{Ir}(1)-\mathrm{C}(23)$ & $2.047(9)$ \\
$\operatorname{Ir}(1)-\mathrm{C}(11)$ & $2.057(9)$ \\
$\operatorname{Ir}(1)-\mathrm{N}(1)$ & $2.082(7)$ \\
$\operatorname{Ir}(1)-\mathrm{N}(2)$ & $2.083(7)$ \\
$\operatorname{Ir}(1)-\mathrm{P}(1)$ & $2.420(2)$ \\
$\operatorname{Ir}(1)-\mathrm{P}(2)$ & $2.431(2)$ \\
$\mathrm{N}(1)-\mathrm{C}(5)$ & $1.334(12)$ \\
$\mathrm{N}(1)-\mathrm{C}(1)$ & $1.339(12)$ \\
$\mathrm{N}(2)-\mathrm{C}(13)$ & $1.324(12)$ \\
$\mathrm{N}(2)-\mathrm{C}(17)$ & $1.363(12)$ \\
$\mathrm{B}(1)-\mathrm{C}(57)$ & $1.631(14)$ \\
$\mathrm{B}(1)-\mathrm{C}(49)$ & $1.664(13)$ \\
$\mathrm{B}(1)-\mathrm{C}(51)$ & $1.651(13)$ \\
$\mathrm{B}(1)-\mathrm{C}(50)$ & $1.667(13)$ \\
$\mathrm{P}(1)-\mathrm{C}(49)$ & $1.809(8)$ \\
$\mathrm{P}(1)-\mathrm{C}(36)$ & $1.852(9)$ \\
$\mathrm{P}(1)-\mathrm{C}(25)$ & $1.860(9)$ \\
$\mathrm{P}(2)-\mathrm{C}(50)$ & $1.823(9)$ \\
$\mathrm{P}(2)-\mathrm{C}(43)$ & $1.841(9)$ \\
$\mathrm{P}(2)-\mathrm{C}(37)$ & $1.848(9)$ \\
$\mathrm{C}(1)-\mathrm{C}(2)$ & $1.380(15)$ \\
$\mathrm{C}(2)-\mathrm{C}(3)$ & $1.410(18)$ \\
$\mathrm{C}(3)-\mathrm{C}(4)$ & $1.341(17)$ \\
$\mathrm{C}(4)-\mathrm{C}(5)$ & $1.419(16)$ \\
$\mathrm{C}(5)-\mathrm{C}(6)$ & $1.435(15)$ \\
$\mathrm{C}(6)-\mathrm{C}(11)$ & $1.360(15)$ \\
$\mathrm{C}(6)-\mathrm{C}(7)$ & $1.460(15)$ \\
\end{tabular}




\begin{tabular}{|c|c|}
\hline$C(7)-C(8)$ & $1.36(2)$ \\
\hline $\mathrm{C}(8)-\mathrm{C}(9)$ & $1.37(2)$ \\
\hline$C(9)-C(10)$ & $1.369(16)$ \\
\hline $\mathrm{C}(9)-\mathrm{C}(12)$ & $1.532(18)$ \\
\hline$C(10)-C(11)$ & $1.403(14)$ \\
\hline$C(13)-C(14)$ & $1.369(14)$ \\
\hline$C(14)-C(15)$ & $1.457(17)$ \\
\hline$C(15)-C(16)$ & $1.317(17)$ \\
\hline$C(16)-C(17)$ & $1.398(14)$ \\
\hline$C(17)-C(18)$ & $1.444(14)$ \\
\hline$C(18)-C(19)$ & $1.412(14)$ \\
\hline$C(18)-C(23)$ & $1.429(13)$ \\
\hline$C(19)-C(20)$ & $1.332(17)$ \\
\hline$C(20)-C(21)$ & $1.399(18)$ \\
\hline$C(21)-C(22)$ & $1.401(15)$ \\
\hline$C(21)-C(24)$ & $1.516(18)$ \\
\hline$C(22)-C(23)$ & $1.438(14)$ \\
\hline$C(25)-C(26)$ & $1.363(13)$ \\
\hline$C(25)-C(30)$ & $1.387(13)$ \\
\hline$C(26)-C(27)$ & $1.365(14)$ \\
\hline $\mathrm{C}(27)-\mathrm{C}(28)$ & $1.368(18)$ \\
\hline$C(28)-C(29)$ & $1.330(17)$ \\
\hline$C(29)-C(30)$ & $1.404(15)$ \\
\hline$C(31)-C(32)$ & $1.371(14)$ \\
\hline$C(31)-C(36)$ & $1.405(12)$ \\
\hline$C(32)-C(33)$ & $1.340(16)$ \\
\hline $\mathrm{C}(33)-\mathrm{C}(34)$ & $1.350(15)$ \\
\hline$C(34)-C(35)$ & $1.368(14)$ \\
\hline$C(35)-C(36)$ & $1.366(12)$ \\
\hline $\mathrm{C}(37)-\mathrm{C}(38)$ & $1.373(15)$ \\
\hline$C(37)-C(42)$ & $1.403(15)$ \\
\hline $\mathrm{C}(38)-\mathrm{C}(39)$ & $1.410(16)$ \\
\hline$C(39)-C(40)$ & $1.37(2)$ \\
\hline$C(40)-C(41)$ & $1.34(2)$ \\
\hline$C(41)-C(42)$ & $1.373(15)$ \\
\hline$C(43)-C(44)$ & $1.378(14)$ \\
\hline
\end{tabular}




\begin{tabular}{ll}
$\mathrm{C}(43)-\mathrm{C}(48)$ & $1.385(13)$ \\
$\mathrm{C}(44)-\mathrm{C}(45)$ & $1.367(15)$ \\
$\mathrm{C}(45)-\mathrm{C}(46)$ & $1.369(17)$ \\
$\mathrm{C}(46)-\mathrm{C}(47)$ & $1.360(18)$ \\
$\mathrm{C}(47)-\mathrm{C}(48)$ & $1.403(16)$ \\
$\mathrm{C}(51)-\mathrm{C}(56)$ & $1.368(13)$ \\
$\mathrm{C}(51)-\mathrm{C}(52)$ & $1.419(13)$ \\
$\mathrm{C}(52)-\mathrm{C}(53)$ & $1.351(14)$ \\
$\mathrm{C}(53)-\mathrm{C}(54)$ & $1.404(16)$ \\
$\mathrm{C}(54)-\mathrm{C}(55)$ & $1.317(16)$ \\
$\mathrm{C}(55)-\mathrm{C}(56)$ & $1.434(14)$ \\
$\mathrm{C}(57)-\mathrm{C}(58)$ & $1.397(14)$ \\
$\mathrm{C}(57)-\mathrm{C}(62)$ & $1.408(13)$ \\
$\mathrm{C}(58)-\mathrm{C}(59)$ & $1.386(18)$ \\
$\mathrm{C}(59)-\mathrm{C}(60)$ & $1.36(2)$ \\
$\mathrm{C}(60)-\mathrm{C}(61)$ & $1.352(19)$ \\
$\mathrm{C}(61)-\mathrm{C}(62)$ & $1.353(15)$ \\
\hline
\end{tabular}

Table S 7. Bond angles $\left[^{\circ}\right]$ for (tpy)2Ir( $\left.\mathrm{PPh} 2 \mathrm{CH} 2\right) 2 \mathrm{BPh} 2 \cdot \mathrm{H} 2 \mathrm{O}$.

\begin{tabular}{lr}
\hline $\mathrm{C}(23)-\operatorname{Ir}(1)-\mathrm{C}(11)$ & $82.0(4)$ \\
$\mathrm{C}(23)-\operatorname{Ir}(1)-\mathrm{N}(1)$ & $92.4(3)$ \\
$\mathrm{C}(11)-\operatorname{Ir}(1)-\mathrm{N}(1)$ & $78.3(4)$ \\
$\mathrm{C}(23)-\operatorname{Ir}(1)-\mathrm{N}(2)$ & $79.4(3)$ \\
$\mathrm{C}(11)-\operatorname{Ir}(1)-\mathrm{N}(2)$ & $93.6(4)$ \\
$\mathrm{N}(1)-\operatorname{Ir}(1)-\mathrm{N}(2)$ & $169.2(3)$ \\
$\mathrm{C}(23)-\operatorname{Ir}(1)-\mathrm{P}(1)$ & $173.4(3)$ \\
$\mathrm{C}(11)-\operatorname{Ir}(1)-\mathrm{P}(1)$ & $92.0(3)$ \\
$\mathrm{N}(1)-\operatorname{Ir}(1)-\mathrm{P}(1)$ & $83.6(2)$ \\
$\mathrm{N}(2)-\operatorname{Ir}(1)-\mathrm{P}(1)$ & $103.9(2)$ \\
$\mathrm{C}(23)-\operatorname{Ir}(1)-\mathrm{P}(2)$ & $94.7(3)$ \\
$\mathrm{C}(11)-\operatorname{Ir}(1)-\mathrm{P}(2)$ & $175.9(3)$ \\
$\mathrm{N}(1)-\operatorname{Ir}(1)-\mathrm{P}(2)$ & $104.4(2)$ \\
$\mathrm{N}(2)-\operatorname{Ir}(1)-\mathrm{P}(2)$ & $83.4(2)$
\end{tabular}




\begin{tabular}{|c|c|}
\hline $\mathrm{P}(1)-\operatorname{Ir}(1)-\mathrm{P}(2)$ & $91.43(8)$ \\
\hline $\mathrm{C}(5)-\mathrm{N}(1)-\mathrm{C}(1)$ & $119.5(9)$ \\
\hline $\mathrm{C}(5)-\mathrm{N}(1)-\operatorname{Ir}(1)$ & $115.7(7)$ \\
\hline $\mathrm{C}(1)-\mathrm{N}(1)-\operatorname{Ir}(1)$ & $124.7(7)$ \\
\hline $\mathrm{C}(13)-\mathrm{N}(2)-\mathrm{C}(17)$ & $118.6(8)$ \\
\hline $\mathrm{C}(13)-\mathrm{N}(2)-\operatorname{Ir}(1)$ & $125.9(6)$ \\
\hline $\mathrm{C}(17)-\mathrm{N}(2)-\operatorname{Ir}(1)$ & $115.5(6)$ \\
\hline $\mathrm{C}(57)-\mathrm{B}(1)-\mathrm{C}(49)$ & 109.2(7) \\
\hline $\mathrm{C}(57)-\mathrm{B}(1)-\mathrm{C}(51)$ & $105.5(7)$ \\
\hline $\mathrm{C}(49)-\mathrm{B}(1)-\mathrm{C}(51)$ & $109.3(7)$ \\
\hline $\mathrm{C}(57)-\mathrm{B}(1)-\mathrm{C}(50)$ & $110.3(7)$ \\
\hline $\mathrm{C}(49)-\mathrm{B}(1)-\mathrm{C}(50)$ & $112.3(7)$ \\
\hline $\mathrm{C}(51)-\mathrm{B}(1)-\mathrm{C}(50)$ & $110.0(7)$ \\
\hline C(49)-P(1)-C(36) & $108.1(4)$ \\
\hline C(49)-P(1)-C(25) & $102.4(4)$ \\
\hline $\mathrm{C}(36)-\mathrm{P}(1)-\mathrm{C}(25)$ & $98.2(4)$ \\
\hline$C(49)-P(1)-\operatorname{Ir}(1)$ & $112.5(3)$ \\
\hline$C(36)-P(1)-\operatorname{Ir}(1)$ & $121.8(3)$ \\
\hline$C(25)-P(1)-\operatorname{Ir}(1)$ & $111.4(3)$ \\
\hline $\mathrm{C}(50)-\mathrm{P}(2)-\mathrm{C}(43)$ & $108.1(4)$ \\
\hline C(50)-P(2)-C(37) & $104.0(4)$ \\
\hline $\mathrm{C}(43)-\mathrm{P}(2)-\mathrm{C}(37)$ & $97.4(4)$ \\
\hline $\mathrm{C}(50)-\mathrm{P}(2)-\operatorname{Ir}(1)$ & $112.8(3)$ \\
\hline $\mathrm{C}(43)-\mathrm{P}(2)-\operatorname{Ir}(1)$ & $121.7(3)$ \\
\hline $\mathrm{C}(37)-\mathrm{P}(2)-\operatorname{Ir}(1)$ & $110.5(3)$ \\
\hline $\mathrm{N}(1)-\mathrm{C}(1)-\mathrm{C}(2)$ & $123.4(11)$ \\
\hline$C(1)-C(2)-C(3)$ & $117.4(11)$ \\
\hline$C(4)-C(3)-C(2)$ & $118.9(11)$ \\
\hline$C(3)-C(4)-C(5)$ & 121.1(12) \\
\hline $\mathrm{N}(1)-\mathrm{C}(5)-\mathrm{C}(6)$ & $114.5(9)$ \\
\hline $\mathrm{N}(1)-\mathrm{C}(5)-\mathrm{C}(4)$ & $119.6(10)$ \\
\hline$C(6)-C(5)-C(4)$ & $125.9(10)$ \\
\hline$C(11)-C(6)-C(5)$ & 117.3(9) \\
\hline$C(11)-C(6)-C(7)$ & $120.9(11)$ \\
\hline$C(5)-C(6)-C(7)$ & $121.6(11)$ \\
\hline$C(8)-C(7)-C(6)$ & $116.8(13)$ \\
\hline
\end{tabular}




\begin{tabular}{|c|c|}
\hline$C(9)-C(8)-C(7)$ & $123.1(12)$ \\
\hline$C(8)-C(9)-C(10)$ & $118.5(13)$ \\
\hline$C(8)-C(9)-C(12)$ & $121.0(13)$ \\
\hline$C(10)-C(9)-C(12)$ & $120.5(14)$ \\
\hline $\mathrm{C}(9)-\mathrm{C}(10)-\mathrm{C}(11)$ & $122.4(12)$ \\
\hline$C(6)-C(11)-C(10)$ & 118.1(10) \\
\hline$C(6)-C(11)-\operatorname{Ir}(1)$ & $113.7(7)$ \\
\hline $\mathrm{C}(10)-\mathrm{C}(11)-\operatorname{Ir}(1)$ & $128.0(8)$ \\
\hline $\mathrm{N}(2)-\mathrm{C}(13)-\mathrm{C}(14)$ & $124.9(10)$ \\
\hline$C(13)-C(14)-C(15)$ & $116.0(11)$ \\
\hline$C(16)-C(15)-C(14)$ & $118.6(10)$ \\
\hline$C(15)-C(16)-C(17)$ & $122.2(11)$ \\
\hline $\mathrm{N}(2)-\mathrm{C}(17)-\mathrm{C}(16)$ & $119.6(10)$ \\
\hline $\mathrm{N}(2)-\mathrm{C}(17)-\mathrm{C}(18)$ & $115.5(8)$ \\
\hline $\mathrm{C}(16)-\mathrm{C}(17)-\mathrm{C}(18)$ & $124.9(10)$ \\
\hline $\mathrm{C}(19)-\mathrm{C}(18)-\mathrm{C}(23)$ & 119.2(10) \\
\hline $\mathrm{C}(19)-\mathrm{C}(18)-\mathrm{C}(17)$ & $125.7(10)$ \\
\hline $\mathrm{C}(23)-\mathrm{C}(18)-\mathrm{C}(17)$ & 115.1(8) \\
\hline $\mathrm{C}(20)-\mathrm{C}(19)-\mathrm{C}(18)$ & $123.1(11)$ \\
\hline$C(19)-C(20)-C(21)$ & $120.3(11)$ \\
\hline$C(20)-C(21)-C(22)$ & $119.4(12)$ \\
\hline $\mathrm{C}(20)-\mathrm{C}(21)-\mathrm{C}(24)$ & $122.5(11)$ \\
\hline$C(22)-C(21)-C(24)$ & $118.1(12)$ \\
\hline $\mathrm{C}(21)-\mathrm{C}(22)-\mathrm{C}(23)$ & $121.5(11)$ \\
\hline$C(18)-C(23)-C(22)$ & $116.5(9)$ \\
\hline$C(18)-C(23)-\operatorname{Ir}(1)$ & $114.3(7)$ \\
\hline$C(22)-C(23)-\operatorname{Ir}(1)$ & $129.2(7)$ \\
\hline$C(26)-C(25)-C(30)$ & 118.6(9) \\
\hline$C(26)-C(25)-P(1)$ & 121.1(8) \\
\hline $\mathrm{C}(30)-\mathrm{C}(25)-\mathrm{P}(1)$ & $120.2(8)$ \\
\hline$C(25)-C(26)-C(27)$ & $120.4(11)$ \\
\hline$C(26)-C(27)-C(28)$ & $121.7(12)$ \\
\hline $\mathrm{C}(29)-\mathrm{C}(28)-\mathrm{C}(27)$ & $118.5(11)$ \\
\hline$C(28)-C(29)-C(30)$ & $121.7(11)$ \\
\hline$C(25)-C(30)-C(29)$ & $119.0(11)$ \\
\hline$C(32)-C(31)-C(36)$ & $118.6(10)$ \\
\hline
\end{tabular}




$\begin{array}{ll}\mathrm{C}(33)-\mathrm{C}(32)-\mathrm{C}(31) & 122.1(10) \\ \mathrm{C}(32)-\mathrm{C}(33)-\mathrm{C}(34) & 119.2(11) \\ \mathrm{C}(33)-\mathrm{C}(34)-\mathrm{C}(35) & 121.3(10) \\ \mathrm{C}(36)-\mathrm{C}(35)-\mathrm{C}(34) & 120.2(9) \\ \mathrm{C}(35)-\mathrm{C}(36)-\mathrm{C}(31) & 118.6(9) \\ \mathrm{C}(35)-\mathrm{C}(36)-\mathrm{P}(1) & 121.4(7) \\ \mathrm{C}(31)-\mathrm{C}(36)-\mathrm{P}(1) & 119.8(7) \\ \mathrm{C}(38)-\mathrm{C}(37)-\mathrm{C}(42) & 119.8(10) \\ \mathrm{C}(38)-\mathrm{C}(37)-\mathrm{P}(2) & 122.0(8) \\ \mathrm{C}(42)-\mathrm{C}(37)-\mathrm{P}(2) & 118.2(8) \\ \mathrm{C}(37)-\mathrm{C}(38)-\mathrm{C}(39) & 121.1(12) \\ \mathrm{C}(40)-\mathrm{C}(39)-\mathrm{C}(38) & 116.9(12) \\ \mathrm{C}(39)-\mathrm{C}(40)-\mathrm{C}(41) & 122.1(12) \\ \mathrm{C}(40)-\mathrm{C}(41)-\mathrm{C}(42) & 121.8(13) \\ \mathrm{C}(41)-\mathrm{C}(42)-\mathrm{C}(37) & 117.9(12) \\ \mathrm{C}(44)-\mathrm{C}(43)-\mathrm{C}(48) & 117.9(9) \\ \mathrm{C}(44)-\mathrm{C}(43)-\mathrm{P}(2) & 120.3(8) \\ \mathrm{C}(48)-\mathrm{C}(43)-\mathrm{P}(2) & 121.6(8) \\ \mathrm{C}(45)-\mathrm{C}(44)-\mathrm{C}(43) & 121.9(11) \\ \mathrm{C}(44)-\mathrm{C}(45)-\mathrm{C}(46) & 120.2(12) \\ \mathrm{C}(47)-\mathrm{C}(46)-\mathrm{C}(45) & 119.6(11) \\ \mathrm{C}(46)-\mathrm{C}(47)-\mathrm{C}(48) & 120.5(12) \\ \mathrm{C}(43)-\mathrm{C}(48)-\mathrm{C}(47) & 119.8(11) \\ \mathrm{B}(1)-\mathrm{C}(49)-\mathrm{P}(1) & 122.8(6) \\ \mathrm{B}(1)-\mathrm{C}(50)-\mathrm{P}(2) & 120.8(6) \\ \mathrm{C}(56)-\mathrm{C}(51)-\mathrm{C}(52) & 115.6(9) \\ \mathrm{C}(56)-\mathrm{C}(51)-\mathrm{B}(1) & 126.5(8) \\ \mathrm{C}(52)-\mathrm{C}(51)-\mathrm{B}(1) & 117.8(8) \\ \mathrm{C}(53)-\mathrm{C}(52)-\mathrm{C}(51) & 122.7(10) \\ \mathrm{C}(52)-\mathrm{C}(53)-\mathrm{C}(54) & 120.7(11) \\ \mathrm{C}(55)-\mathrm{C}(54)-\mathrm{C}(53) & 118.1(10) \\ \mathrm{C}(54)-\mathrm{C}(55)-\mathrm{C}(56) & \end{array}$




$\begin{array}{ll}\mathrm{C}(59)-\mathrm{C}(58)-\mathrm{C}(57) & 120.9(12) \\ \mathrm{C}(60)-\mathrm{C}(59)-\mathrm{C}(58) & 122.6(13) \\ \mathrm{C}(59)-\mathrm{C}(60)-\mathrm{C}(61) & 117.5(12) \\ \mathrm{C}(62)-\mathrm{C}(61)-\mathrm{C}(60) & 121.1(11) \\ \mathrm{C}(61)-\mathrm{C}(62)-\mathrm{C}(57) & 124.0(11)\end{array}$

Symmetry transformations used to generate equivalent atoms:

Table S 8. Anisotropic displacement parameters $(\AA 2 x 103)$ for (tpy)2Ir(PPh2CH2)2BPh2 $\cdot \mathrm{H} 2 \mathrm{O}$.. The anisotropic displacement factor exponent takes the form: $-2 \pi 2[\mathrm{~h} 2 \mathrm{a} * 2 \mathrm{U} 11+\ldots+2 \mathrm{hka}$ *b*U12]

\begin{tabular}{|c|c|c|c|c|c|c|}
\hline & $U^{11}$ & $\mathrm{U}^{22}$ & $U^{33}$ & $U^{23}$ & $U^{13}$ & $U^{12}$ \\
\hline $\operatorname{Ir}(1)$ & $31(1)$ & $33(1)$ & $31(1)$ & $-3(1)$ & $9(1)$ & $-1(1)$ \\
\hline $\mathrm{N}(1)$ & $29(4)$ & $36(4)$ & $42(4)$ & $0(3)$ & $10(3)$ & $4(3)$ \\
\hline $\mathrm{N}(2)$ & $36(4)$ & $44(5)$ & $37(4)$ & $-10(3)$ & $15(3)$ & $0(4)$ \\
\hline $\mathrm{B}(1)$ & $32(5)$ & $37(6)$ & $29(5)$ & $2(4)$ & $9(4)$ & $5(4)$ \\
\hline $\mathrm{P}(1)$ & $30(1)$ & $33(1)$ & $32(1)$ & $-4(1)$ & $8(1)$ & $-1(1)$ \\
\hline $\mathrm{P}(2)$ & $31(1)$ & $36(1)$ & $33(1)$ & $-4(1)$ & $10(1)$ & $-2(1)$ \\
\hline $\mathrm{C}(1)$ & $50(6)$ & $46(6)$ & $50(6)$ & $3(5)$ & $13(5)$ & $3(5)$ \\
\hline $\mathrm{C}(2)$ & $67(8)$ & $59(7)$ & $89(9)$ & $-8(6)$ & $40(7)$ & $18(6)$ \\
\hline$C(3)$ & $53(8)$ & $105(11)$ & $96(10)$ & $11(9)$ & $28(8)$ & $38(8)$ \\
\hline$C(4)$ & $55(7)$ & 71(9) & $76(8)$ & $12(7)$ & $1(6)$ & $16(6)$ \\
\hline$C(5)$ & $40(6)$ & $49(6)$ & $55(6)$ & $13(5)$ & $4(5)$ & $1(5)$ \\
\hline$C(6)$ & $49(6)$ & $53(6)$ & $42(5)$ & $8(5)$ & $2(5)$ & $-7(5)$ \\
\hline$C(7)$ & $61(8)$ & $125(13)$ & $47(7)$ & $11(8)$ & $-15(6)$ & $-6(8)$ \\
\hline $\mathrm{C}(8)$ & $93(12)$ & $119(12)$ & $36(7)$ & $-2(7)$ & $-10(7)$ & $-21(9)$ \\
\hline$C(9)$ & $73(8)$ & $87(9)$ & $49(8)$ & $-9(7)$ & $12(7)$ & $-26(7)$ \\
\hline$C(10)$ & $66(7)$ & $54(6)$ & $43(5)$ & $-7(5)$ & $18(5)$ & $-16(6)$ \\
\hline$C(11)$ & $43(6)$ & $49(6)$ & $28(5)$ & $-3(4)$ & $4(4)$ & $-12(4)$ \\
\hline$C(12)$ & $137(16)$ & $138(15)$ & $50(7)$ & $-44(8)$ & $23(9)$ & $-20(12)$ \\
\hline$C(13)$ & $40(5)$ & $47(6)$ & $42(5)$ & $-4(4)$ & $14(4)$ & $0(5)$ \\
\hline$C(14)$ & $48(6)$ & $61(7)$ & $62(7)$ & $-10(5)$ & $15(5)$ & $13(5)$ \\
\hline$C(15)$ & $55(8)$ & $89(10)$ & $81(9)$ & $-37(8)$ & $26(7)$ & $11(7)$ \\
\hline$C(16)$ & $51(7)$ & $70(9)$ & $72(8)$ & $-3(6)$ & $36(6)$ & $-4(6)$ \\
\hline
\end{tabular}




\begin{tabular}{|c|c|c|c|c|c|c|}
\hline$C(17)$ & $43(6)$ & $54(6)$ & $36(5)$ & $-7(4)$ & $15(4)$ & $-2(4)$ \\
\hline$C(18)$ & $59(7)$ & $51(6)$ & $34(5)$ & $-5(4)$ & $19(5)$ & $-10(5)$ \\
\hline$C(19)$ & $77(8)$ & $68(8)$ & $58(7)$ & $4(6)$ & $41(6)$ & $-10(7)$ \\
\hline$C(20)$ & $109(12)$ & $69(9)$ & $59(8)$ & $13(6)$ & $42(8)$ & $-22(8)$ \\
\hline$C(21)$ & $87(9)$ & $54(7)$ & $51(7)$ & $3(5)$ & $7(6)$ & $-15(6)$ \\
\hline$C(22)$ & $64(8)$ & $51(6)$ & $40(5)$ & $3(5)$ & $5(5)$ & $-5(6)$ \\
\hline$C(23)$ & $39(5)$ & $42(5)$ & $33(5)$ & $-1(4)$ & $9(4)$ & $0(4)$ \\
\hline$C(24)$ & $137(14)$ & $57(9)$ & $78(9)$ & $17(7)$ & $22(9)$ & $-15(9)$ \\
\hline$C(25)$ & $32(5)$ & $34(5)$ & $45(5)$ & $8(4)$ & $6(4)$ & $-7(4)$ \\
\hline$C(26)$ & $38(5)$ & $57(7)$ & $54(6)$ & $0(5)$ & $14(5)$ & $2(5)$ \\
\hline$C(27)$ & $34(6)$ & $120(11)$ & $66(8)$ & $19(7)$ & $12(6)$ & $12(7)$ \\
\hline$C(28)$ & $37(6)$ & $96(11)$ & $70(9)$ & $15(8)$ & $9(6)$ & $-6(6)$ \\
\hline$C(29)$ & $58(8)$ & $80(9)$ & $44(6)$ & $3(6)$ & $-8(6)$ & $-23(7)$ \\
\hline$C(30)$ & $48(6)$ & $62(7)$ & $48(6)$ & $-6(5)$ & $3(5)$ & $-5(5)$ \\
\hline$C(31)$ & $53(6)$ & $43(6)$ & $54(6)$ & $-10(5)$ & $17(5)$ & $-4(5)$ \\
\hline$C(32)$ & $74(8)$ & $51(7)$ & $62(7)$ & $-24(6)$ & $27(6)$ & $-2(6)$ \\
\hline$C(33)$ & $76(8)$ & $34(6)$ & $68(7)$ & $-6(5)$ & $23(6)$ & $2(6)$ \\
\hline$C(34)$ & $53(6)$ & $45(6)$ & $62(7)$ & $4(5)$ & $18(5)$ & $-4(5)$ \\
\hline$C(35)$ & $36(5)$ & $33(5)$ & $49(6)$ & $5(4)$ & $6(4)$ & $0(4)$ \\
\hline$C(36)$ & $31(5)$ & $35(5)$ & $47(5)$ & $-5(4)$ & $13(4)$ & $-4(4)$ \\
\hline$C(37)$ & $30(5)$ & $59(7)$ & $34(5)$ & $-12(4)$ & $9(4)$ & $-8(5)$ \\
\hline$C(38)$ & $37(6)$ & $70(8)$ & $77(8)$ & $5(6)$ & $21(6)$ & $2(5)$ \\
\hline$C(39)$ & $39(7)$ & $121(13)$ & $96(11)$ & $36(9)$ & $33(7)$ & $18(7)$ \\
\hline $\mathrm{C}(40)$ & $38(7)$ & $160(16)$ & $76(9)$ & $-16(10)$ & $21(7)$ & $-18(9)$ \\
\hline $\mathrm{C}(41)$ & $54(7)$ & $85(9)$ & $79(8)$ & $-8(7)$ & $33(7)$ & $-29(7)$ \\
\hline$C(42)$ & $46(6)$ & $59(7)$ & $52(6)$ & $-4(5)$ & $15(5)$ & $-15(5)$ \\
\hline $\mathrm{C}(43)$ & $42(5)$ & $37(5)$ & $46(5)$ & $-9(4)$ & $15(4)$ & $-6(4)$ \\
\hline $\mathrm{C}(44)$ & $58(7)$ & $49(6)$ & $52(6)$ & $0(5)$ & $14(5)$ & $3(5)$ \\
\hline$C(45)$ & $62(8)$ & $41(6)$ & $86(9)$ & $-14(6)$ & $22(7)$ & $4(5)$ \\
\hline $\mathrm{C}(46)$ & $67(8)$ & $50(7)$ & $105(11)$ & $-30(7)$ & $42(8)$ & $-16(6)$ \\
\hline $\mathrm{C}(47)$ & $69(8)$ & $79(9)$ & $61(7)$ & $-24(7)$ & $28(6)$ & $-28(7)$ \\
\hline C(48) & $48(6)$ & $56(7)$ & $38(5)$ & $-9(4)$ & $13(4)$ & $-20(5)$ \\
\hline C(49) & $35(5)$ & $35(5)$ & $33(4)$ & $-4(4)$ & $14(4)$ & $2(4)$ \\
\hline$C(50)$ & $34(5)$ & $38(5)$ & $27(4)$ & $-5(3)$ & $3(4)$ & $2(4)$ \\
\hline$C(51)$ & $32(5)$ & $36(5)$ & $36(5)$ & $2(4)$ & 11(4) & $-4(4)$ \\
\hline$C(52)$ & $52(6)$ & $45(5)$ & $27(4)$ & $-4(4)$ & $-2(4)$ & $-1(5)$ \\
\hline
\end{tabular}




\begin{tabular}{lcccccc}
$\mathrm{C}(53)$ & $65(8)$ & $52(7)$ & $48(6)$ & $-4(5)$ & $3(6)$ & $-6(6)$ \\
$\mathrm{C}(54)$ & $71(8)$ & $58(7)$ & $38(5)$ & $-16(5)$ & $13(5)$ & $-7(6)$ \\
$\mathrm{C}(55)$ & $55(7)$ & $52(7)$ & $73(8)$ & $-16(6)$ & $23(6)$ & $11(5)$ \\
$\mathrm{C}(56)$ & $38(5)$ & $49(6)$ & $49(6)$ & $-5(5)$ & $10(4)$ & $4(5)$ \\
$\mathrm{C}(57)$ & $43(5)$ & $43(6)$ & $32(5)$ & $-4(4)$ & $9(4)$ & $0(4)$ \\
$\mathrm{C}(58)$ & $69(8)$ & $47(7)$ & $79(8)$ & $8(6)$ & $29(7)$ & $14(6)$ \\
$\mathrm{C}(59)$ & $99(11)$ & $80(10)$ & $91(10)$ & $19(8)$ & $10(9)$ & $53(9)$ \\
$\mathrm{C}(60)$ & $126(14)$ & $48(8)$ & $78(9)$ & $18(7)$ & $37(9)$ & $0(8)$ \\
$\mathrm{C}(61)$ & $79(9)$ & $51(7)$ & $64(7)$ & $5(6)$ & $31(6)$ & $-10(6)$ \\
$\mathrm{C}(62)$ & $58(6)$ & $46(6)$ & $46(6)$ & $9(5)$ & $16(5)$ & $6(5)$ \\
$\mathrm{O}(1)$ & $30(4)$ & $287(12)$ & $29(4)$ & $-33(5)$ & $10(3)$ & $27(6)$ \\
\hline
\end{tabular}

Table S 9. Atomic coordinates ( x 104) and equivalent isotropic displacement parameters ( $\AA 2$ x 103) for $[($ tpy $) 2 \operatorname{Ir}(\mathrm{CN}-\mathrm{t}-\mathrm{Bu}) 2](\mathrm{CF} 3 \mathrm{SO} 3) \cdot \mathrm{CHCl3}$. U(eq) is defined as one third of the trace of the orthogonalized Uij tensor.

\begin{tabular}{|c|c|c|c|c|}
\hline & $\mathrm{x}$ & $\mathrm{y}$ & $\mathrm{z}$ & $\mathrm{U}(\mathrm{eq})$ \\
\hline $\operatorname{Ir}(1)$ & $5231(1)$ & $1788(1)$ & $7549(1)$ & $30(1)$ \\
\hline $\mathrm{Cl}(1)$ & $1204(4)$ & $748(5)$ & $8218(2)$ & 251(3) \\
\hline $\mathrm{Cl}(2)$ & 393(4) & 2633(4) & $8336(3)$ & $223(2)$ \\
\hline $\mathrm{Cl}(3)$ & $-872(3)$ & $1016(4)$ & $8041(2)$ & $197(2)$ \\
\hline $\mathrm{S}(1)$ & $5915(1)$ & $6118(1)$ & 8711(1) & $59(1)$ \\
\hline $\mathrm{F}(1)$ & $6422(4)$ & $5901(5)$ & $9835(2)$ & $119(2)$ \\
\hline $\mathrm{F}(2)$ & $4895(4)$ & $5879(4)$ & $9560(2)$ & $99(2)$ \\
\hline $\mathrm{F}(3)$ & $5686(5)$ & $7280(4)$ & $9594(2)$ & $100(2)$ \\
\hline $\mathrm{O}(1)$ & $5925(4)$ & $5051(3)$ & $8658(2)$ & $75(2)$ \\
\hline $\mathrm{O}(2)$ & $5047(5)$ & $6609(4)$ & $8378(3)$ & $86(2)$ \\
\hline $\mathrm{O}(3)$ & $6794(4)$ & $6643(4)$ & $8652(3)$ & $83(2)$ \\
\hline $\mathrm{N}(1)$ & $4142(3)$ & $2856(3)$ & $7574(2)$ & $39(1)$ \\
\hline $\mathrm{N}(2)$ & $6087(3)$ & $515(3)$ & $7522(2)$ & $31(1)$ \\
\hline $\mathrm{N}(3)$ & $6682(3)$ & 2483(3) & $8676(2)$ & $41(1)$ \\
\hline $\mathrm{N}(4)$ & $5845(3)$ & $2869(3)$ & $6438(2)$ & $41(1)$ \\
\hline $\mathrm{C}(1)$ & $4230(5)$ & $3656(5)$ & $7949(3)$ & $57(2)$ \\
\hline$C(2)$ & $3459(7)$ & 4291(6) & 7984(4) & $83(3)$ \\
\hline
\end{tabular}




\begin{tabular}{|c|c|c|c|c|}
\hline$C(3)$ & $2569(6)$ & $4123(6)$ & $7633(4)$ & $84(3)$ \\
\hline$C(4)$ & $2467(6)$ & $3316(5)$ & $7249(4)$ & $70(2)$ \\
\hline$C(5)$ & $3266(4)$ & 2677(4) & $7221(3)$ & $44(1)$ \\
\hline$C(6)$ & $3275(4)$ & 1819(4) & $6833(2)$ & $38(1)$ \\
\hline$C(7)$ & 2497(4) & 1574(5) & $6384(3)$ & $49(2)$ \\
\hline $\mathrm{C}(8)$ & $2596(4)$ & $787(5)$ & $6004(3)$ & $53(2)$ \\
\hline$C(9)$ & $3472(5)$ & $229(5)$ & $6057(3)$ & $50(2)$ \\
\hline$C(10)$ & $4239(4)$ & $465(4)$ & $6516(2)$ & $41(1)$ \\
\hline$C(11)$ & $4164(4)$ & $1246(4)$ & $6904(2)$ & $35(1)$ \\
\hline$C(12)$ & $3587(6)$ & $-614(6)$ & $5632(3)$ & $84(3)$ \\
\hline$C(13)$ & $6801(4)$ & $394(5)$ & $7175(2)$ & $44(1)$ \\
\hline$C(14)$ & $7307(4)$ & $-497(5)$ & $7146(3)$ & $53(2)$ \\
\hline$C(15)$ & $7068(5)$ & $-1302(5)$ & $7478(3)$ & $60(2)$ \\
\hline$C(16)$ & $6361(5)$ & $-1200(5)$ & $7833(3)$ & $54(2)$ \\
\hline$C(17)$ & $5874(4)$ & $-272(4)$ & $7860(2)$ & $38(1)$ \\
\hline$C(18)$ & $5129(4)$ & $-51(4)$ & $8241(2)$ & $39(1)$ \\
\hline$C(19)$ & $4883(5)$ & $-728(5)$ & $8659(3)$ & $56(2)$ \\
\hline$C(20)$ & $4238(6)$ & $-441(6)$ & 9031(3) & $65(2)$ \\
\hline$C(21)$ & $3812(5)$ & $512(5)$ & 8994(3) & $58(2)$ \\
\hline$C(22)$ & $4036(4)$ & $1170(5)$ & $8557(2)$ & $45(1)$ \\
\hline$C(23)$ & $4697(4)$ & $894(4)$ & $8172(2)$ & $35(1)$ \\
\hline$C(24)$ & $3133(7)$ & $839(7)$ & $9425(4)$ & $91(3)$ \\
\hline$C(25)$ & $6178(4)$ & 2295(4) & $8238(2)$ & $37(1)$ \\
\hline$C(26)$ & $7128(5)$ & 2554(5) & $9296(3)$ & $54(2)$ \\
\hline$C(27)$ & $7917(6)$ & $3361(6)$ & $9364(3)$ & $75(2)$ \\
\hline$C(28)$ & $6295(7)$ & $2815(7)$ & $9633(3)$ & $81(2)$ \\
\hline$C(29)$ & $7546(7)$ & $1518(6)$ & $9463(4)$ & $88(3)$ \\
\hline$C(30)$ & $5697(4)$ & 2519(4) & $6871(2)$ & $37(1)$ \\
\hline$C(31)$ & $5864(6)$ & $3203(5)$ & $5834(3)$ & $55(2)$ \\
\hline$C(32)$ & $4937(7)$ & $3824(8)$ & $5656(4)$ & $104(3)$ \\
\hline$C(33)$ & $5875(8)$ & $2258(6)$ & $5465(3)$ & $93(3)$ \\
\hline$C(34)$ & $6800(6)$ & 3813(6) & $5826(3)$ & $80(2)$ \\
\hline$C(35)$ & $236(7)$ & $1524(8)$ & 7971(4) & $96(3)$ \\
\hline$C(36)$ & $5734(5)$ & $6322(6)$ & $9463(3)$ & $61(2)$ \\
\hline
\end{tabular}


Table S 10. Bond lengths $[\AA]$ and angles $\left[{ }^{\circ}\right]$ for $[(\operatorname{tpy}) 2 \operatorname{Ir}(\mathrm{CN}-\mathrm{t}-\mathrm{Bu}) 2](\mathrm{CF} 3 \mathrm{SO} 3) \cdot \mathrm{CHCl} 3$.

\begin{tabular}{|c|c|}
\hline $\operatorname{Ir}(1)-C(25)$ & $2.004(6)$ \\
\hline $\operatorname{Ir}(1)-C(30)$ & $2.018(6)$ \\
\hline $\operatorname{Ir}(1)-C(11)$ & $2.047(5)$ \\
\hline $\operatorname{Ir}(1)-\mathrm{N}(2)$ & $2.059(4)$ \\
\hline $\operatorname{Ir}(1)-\mathrm{N}(1)$ & $2.061(4)$ \\
\hline $\operatorname{Ir}(1)-C(23)$ & $2.072(5)$ \\
\hline $\mathrm{Cl}(1)-\mathrm{C}(35)$ & $1.704(11)$ \\
\hline $\mathrm{Cl}(2)-\mathrm{C}(35)$ & $1.691(11)$ \\
\hline $\mathrm{Cl}(3)-\mathrm{C}(35)$ & $1.686(10)$ \\
\hline $\mathrm{S}(1)-\mathrm{O}(3)$ & $1.412(6)$ \\
\hline $\mathrm{S}(1)-\mathrm{O}(1)$ & $1.421(5)$ \\
\hline $\mathrm{S}(1)-\mathrm{O}(2)$ & $1.463(6)$ \\
\hline$S(1)-C(36)$ & $1.803(8)$ \\
\hline $\mathrm{F}(1)-\mathrm{C}(36)$ & $1.299(8)$ \\
\hline $\mathrm{F}(2)-\mathrm{C}(36)$ & $1.336(8)$ \\
\hline $\mathrm{F}(3)-\mathrm{C}(36)$ & $1.310(8)$ \\
\hline $\mathrm{N}(1)-\mathrm{C}(1)$ & $1.359(7)$ \\
\hline $\mathrm{N}(1)-\mathrm{C}(5)$ & $1.362(7)$ \\
\hline $\mathrm{N}(2)-\mathrm{C}(13)$ & $1.359(6)$ \\
\hline $\mathrm{N}(2)-\mathrm{C}(17)$ & $1.359(6)$ \\
\hline $\mathrm{N}(3)-\mathrm{C}(25)$ & $1.158(7)$ \\
\hline $\mathrm{N}(3)-\mathrm{C}(26)$ & $1.464(7)$ \\
\hline $\mathrm{N}(4)-\mathrm{C}(30)$ & $1.143(7)$ \\
\hline $\mathrm{N}(4)-\mathrm{C}(31)$ & $1.461(7)$ \\
\hline $\mathrm{C}(1)-\mathrm{C}(2)$ & $1.360(9)$ \\
\hline $\mathrm{C}(2)-\mathrm{C}(3)$ & $1.372(12)$ \\
\hline $\mathrm{C}(3)-\mathrm{C}(4)$ & $1.382(10)$ \\
\hline$C(4)-C(5)$ & $1.392(8)$ \\
\hline$C(5)-C(6)$ & $1.446(8)$ \\
\hline $\mathrm{C}(6)-\mathrm{C}(7)$ & $1.405(8)$ \\
\hline$C(6)-C(11)$ & $1.420(7)$ \\
\hline $\mathrm{C}(7)-\mathrm{C}(8)$ & $1.380(9)$ \\
\hline $\mathrm{C}(8)-\mathrm{C}(9)$ & $1.397(9)$ \\
\hline $\mathrm{C}(9)-\mathrm{C}(10)$ & $1.405(7)$ \\
\hline
\end{tabular}




$\begin{array}{ll}\mathrm{C}(9)-\mathrm{C}(12) & 1.508(9) \\ \mathrm{C}(10)-\mathrm{C}(11) & 1.382(7) \\ \mathrm{C}(13)-\mathrm{C}(14) & 1.375(8) \\ \mathrm{C}(14)-\mathrm{C}(15) & 1.380(9) \\ \mathrm{C}(15)-\mathrm{C}(16) & 1.361(9) \\ \mathrm{C}(16)-\mathrm{C}(17) & 1.405(8) \\ \mathrm{C}(17)-\mathrm{C}(18) & 1.466(7) \\ \mathrm{C}(18)-\mathrm{C}(23) & 1.385(7) \\ \mathrm{C}(18)-\mathrm{C}(19) & 1.393(8) \\ \mathrm{C}(19)-\mathrm{C}(20) & 1.370(9) \\ \mathrm{C}(20)-\mathrm{C}(21) & 1.389(9) \\ \mathrm{C}(21)-\mathrm{C}(22) & 1.397(8) \\ \mathrm{C}(21)-\mathrm{C}(24) & 1.519(9) \\ \mathrm{C}(22)-\mathrm{C}(23) & 1.404(7) \\ \mathrm{C}(26)-\mathrm{C}(27) & 1.509(9) \\ \mathrm{C}(26)-\mathrm{C}(28) & 1.510(10) \\ \mathrm{C}(26)-\mathrm{C}(29) & 1.514(10) \\ \mathrm{C}(31)-\mathrm{C}(32) & 1.514(11) \\ \mathrm{C}(31)-\mathrm{C}(34) & 1.515(10) \\ \mathrm{C}(31)-\mathrm{C}(33) & 1.516(9)\end{array}$

Table S 11. Bond angles $\left[^{\circ}\right]$ for $[($ tpy $) 2 \operatorname{Ir}(\mathrm{CN}-\mathrm{t}-\mathrm{Bu}) 2](\mathrm{CF} 3 \mathrm{SO} 3) \cdot \mathrm{CHCl3}$.

$\begin{array}{lc}\mathrm{C}(25)-\operatorname{Ir}(1)-\mathrm{C}(30) & 102.0(2) \\ \mathrm{C}(25)-\operatorname{Ir}(1)-\mathrm{C}(11) & 174.1(2) \\ \mathrm{C}(30)-\operatorname{Ir}(1)-\mathrm{C}(11) & 83.2(2) \\ \mathrm{C}(25)-\operatorname{Ir}(1)-\mathrm{N}(2) & 89.84(19) \\ \mathrm{C}(30)-\operatorname{Ir}(1)-\mathrm{N}(2) & 97.11(18) \\ \mathrm{C}(11)-\operatorname{Ir}(1)-\mathrm{N}(2) & 92.07(18) \\ \mathrm{C}(25)-\operatorname{Ir}(1)-\mathrm{N}(1) & 97.5(2) \\ \mathrm{C}(30)-\operatorname{Ir}(1)-\mathrm{N}(1) & 90.22(19) \\ \mathrm{C}(11)-\operatorname{Ir}(1)-\mathrm{N}(1) & 79.82(19) \\ \mathrm{N}(2)-\operatorname{Ir}(1)-\mathrm{N}(1) & 168.38(17)\end{array}$




\begin{tabular}{|c|c|}
\hline$C(25)-\operatorname{Ir}(1)-C(23)$ & $84.2(2)$ \\
\hline$C(30)-\operatorname{Ir}(1)-C(23)$ & $172.8(2)$ \\
\hline$C(11)-\operatorname{Ir}(1)-C(23)$ & $90.7(2)$ \\
\hline $\mathrm{N}(2)-\operatorname{Ir}(1)-\mathrm{C}(23)$ & $79.20(18)$ \\
\hline $\mathrm{N}(1)-\operatorname{Ir}(1)-\mathrm{C}(23)$ & $92.51(19)$ \\
\hline $\mathrm{O}(3)-\mathrm{S}(1)-\mathrm{O}(1)$ & $117.7(3)$ \\
\hline $\mathrm{O}(3)-\mathrm{S}(1)-\mathrm{O}(2)$ & $111.4(4)$ \\
\hline $\mathrm{O}(1)-\mathrm{S}(1)-\mathrm{O}(2)$ & $114.6(4)$ \\
\hline $\mathrm{O}(3)-\mathrm{S}(1)-\mathrm{C}(36)$ & $105.0(4)$ \\
\hline $\mathrm{O}(1)-\mathrm{S}(1)-\mathrm{C}(36)$ & $103.6(3)$ \\
\hline $\mathrm{O}(2)-\mathrm{S}(1)-\mathrm{C}(36)$ & $102.5(3)$ \\
\hline$C(1)-N(1)-C(5)$ & $120.0(5)$ \\
\hline$C(1)-N(1)-\operatorname{Ir}(1)$ & $124.0(4)$ \\
\hline$C(5)-N(1)-\operatorname{Ir}(1)$ & $115.8(4)$ \\
\hline $\mathrm{C}(13)-\mathrm{N}(2)-\mathrm{C}(17)$ & $118.6(5)$ \\
\hline $\mathrm{C}(13)-\mathrm{N}(2)-\operatorname{Ir}(1)$ & $125.1(4)$ \\
\hline $\mathrm{C}(17)-\mathrm{N}(2)-\operatorname{Ir}(1)$ & $116.2(3)$ \\
\hline $\mathrm{C}(25)-\mathrm{N}(3)-\mathrm{C}(26)$ & $164.8(6)$ \\
\hline $\mathrm{C}(30)-\mathrm{N}(4)-\mathrm{C}(31)$ & $168.8(6)$ \\
\hline $\mathrm{N}(1)-\mathrm{C}(1)-\mathrm{C}(2)$ & $121.7(7)$ \\
\hline$C(1)-C(2)-C(3)$ & 119.3(7) \\
\hline$C(2)-C(3)-C(4)$ & $119.8(7)$ \\
\hline$C(3)-C(4)-C(5)$ & $119.8(7)$ \\
\hline $\mathrm{N}(1)-\mathrm{C}(5)-\mathrm{C}(4)$ & $119.4(6)$ \\
\hline $\mathrm{N}(1)-\mathrm{C}(5)-\mathrm{C}(6)$ & $114.7(5)$ \\
\hline$C(4)-C(5)-C(6)$ & $125.9(6)$ \\
\hline$C(7)-C(6)-C(11)$ & $119.9(5)$ \\
\hline$C(7)-C(6)-C(5)$ & $124.1(5)$ \\
\hline $\mathrm{C}(11)-\mathrm{C}(6)-\mathrm{C}(5)$ & $115.9(5)$ \\
\hline$C(8)-C(7)-C(6)$ & $120.4(6)$ \\
\hline $\mathrm{C}(7)-\mathrm{C}(8)-\mathrm{C}(9)$ & $120.6(5)$ \\
\hline $\mathrm{C}(8)-\mathrm{C}(9)-\mathrm{C}(10)$ & $118.7(6)$ \\
\hline $\mathrm{C}(8)-\mathrm{C}(9)-\mathrm{C}(12)$ & $120.5(6)$ \\
\hline $\mathrm{C}(10)-\mathrm{C}(9)-\mathrm{C}(12)$ & $120.8(6)$ \\
\hline $\mathrm{C}(11)-\mathrm{C}(10)-\mathrm{C}(9)$ & $122.2(5)$ \\
\hline$C(10)-C(11)-C(6)$ & $118.2(5)$ \\
\hline
\end{tabular}




\begin{tabular}{|c|c|}
\hline$C(10)-C(11)-\operatorname{Ir}(1)$ & $128.1(4)$ \\
\hline$C(6)-C(11)-\operatorname{Ir}(1)$ & $113.5(4)$ \\
\hline $\mathrm{N}(2)-\mathrm{C}(13)-\mathrm{C}(14)$ & $122.7(5)$ \\
\hline $\mathrm{C}(13)-\mathrm{C}(14)-\mathrm{C}(15)$ & $118.4(6)$ \\
\hline$C(16)-C(15)-C(14)$ & $120.2(6)$ \\
\hline$C(15)-C(16)-C(17)$ & $119.8(6)$ \\
\hline $\mathrm{N}(2)-\mathrm{C}(17)-\mathrm{C}(16)$ & $120.3(5)$ \\
\hline $\mathrm{N}(2)-\mathrm{C}(17)-\mathrm{C}(18)$ & $114.5(5)$ \\
\hline $\mathrm{C}(16)-\mathrm{C}(17)-\mathrm{C}(18)$ & $125.2(5)$ \\
\hline $\mathrm{C}(23)-\mathrm{C}(18)-\mathrm{C}(19)$ & $121.3(5)$ \\
\hline $\mathrm{C}(23)-\mathrm{C}(18)-\mathrm{C}(17)$ & $115.7(5)$ \\
\hline $\mathrm{C}(19)-\mathrm{C}(18)-\mathrm{C}(17)$ & $122.9(5)$ \\
\hline $\mathrm{C}(20)-\mathrm{C}(19)-\mathrm{C}(18)$ & 119.7(6) \\
\hline$C(19)-C(20)-C(21)$ & 121.1(6) \\
\hline $\mathrm{C}(20)-\mathrm{C}(21)-\mathrm{C}(22)$ & $118.6(6)$ \\
\hline$C(20)-C(21)-C(24)$ & $120.7(6)$ \\
\hline $\mathrm{C}(22)-\mathrm{C}(21)-\mathrm{C}(24)$ & $120.7(7)$ \\
\hline $\mathrm{C}(21)-\mathrm{C}(22)-\mathrm{C}(23)$ & $121.4(6)$ \\
\hline $\mathrm{C}(18)-\mathrm{C}(23)-\mathrm{C}(22)$ & $117.8(5)$ \\
\hline$C(18)-C(23)-\operatorname{Ir}(1)$ & 114.1(4) \\
\hline$C(22)-C(23)-\operatorname{Ir}(1)$ & $127.8(4)$ \\
\hline $\mathrm{N}(3)-\mathrm{C}(25)-\operatorname{Ir}(1)$ & $171.0(5)$ \\
\hline $\mathrm{N}(3)-\mathrm{C}(26)-\mathrm{C}(27)$ & $109.5(5)$ \\
\hline $\mathrm{N}(3)-\mathrm{C}(26)-\mathrm{C}(28)$ & 106.1(5) \\
\hline$C(27)-C(26)-C(28)$ & $111.4(6)$ \\
\hline $\mathrm{N}(3)-\mathrm{C}(26)-\mathrm{C}(29)$ & $106.0(5)$ \\
\hline$C(27)-C(26)-C(29)$ & $112.4(7)$ \\
\hline$C(28)-C(26)-C(29)$ & 111.2(7) \\
\hline $\mathrm{N}(4)-\mathrm{C}(30)-\operatorname{Ir}(1)$ & $170.0(5)$ \\
\hline $\mathrm{N}(4)-\mathrm{C}(31)-\mathrm{C}(32)$ & $106.8(6)$ \\
\hline $\mathrm{N}(4)-\mathrm{C}(31)-\mathrm{C}(34)$ & $108.0(6)$ \\
\hline $\mathrm{C}(32)-\mathrm{C}(31)-\mathrm{C}(34)$ & $112.3(7)$ \\
\hline $\mathrm{N}(4)-\mathrm{C}(31)-\mathrm{C}(33)$ & $106.5(5)$ \\
\hline $\mathrm{C}(32)-\mathrm{C}(31)-\mathrm{C}(33)$ & 112.1(7) \\
\hline $\mathrm{C}(34)-\mathrm{C}(31)-\mathrm{C}(33)$ & $110.9(7)$ \\
\hline $\mathrm{Cl}(3)-\mathrm{C}(35)-\mathrm{Cl}(2)$ & $110.5(6)$ \\
\hline
\end{tabular}




$\begin{array}{ll}\mathrm{Cl}(3)-\mathrm{C}(35)-\mathrm{Cl}(1) & 112.9(7) \\ \mathrm{Cl}(2)-\mathrm{C}(35)-\mathrm{Cl}(1) & 109.0(6) \\ \mathrm{F}(1)-\mathrm{C}(36)-\mathrm{F}(3) & 108.7(6) \\ \mathrm{F}(1)-\mathrm{C}(36)-\mathrm{F}(2) & 104.7(6) \\ \mathrm{F}(3)-\mathrm{C}(36)-\mathrm{F}(2) & 108.3(6) \\ \mathrm{F}(1)-\mathrm{C}(36)-\mathrm{S}(1) & 112.2(6) \\ \mathrm{F}(3)-\mathrm{C}(36)-\mathrm{S}(1) & 112.7(5) \\ \mathrm{F}(2)-\mathrm{C}(36)-\mathrm{S}(1) & 109.8(5)\end{array}$

Symmetry transformations used to generate equivalent atoms:

Table S 12. Anisotropic displacement parameters $(\AA 2 x 103)$ for $[(\operatorname{tpy}) 2 \operatorname{Ir}(\mathrm{CN}-\mathrm{t}-\mathrm{Bu}) 2](\mathrm{CF} 3 \mathrm{SO} 3) \cdot \mathrm{CHCl} 3$.

The anisotropic displacement factor exponent takes the form: $-2 \pi 2\left[\mathrm{~h} 2 \mathrm{a} * 2 \mathrm{U} 11+\ldots+2 \mathrm{hka} \mathrm{b}^{*} \mathrm{U} 12\right]$.

\begin{tabular}{|c|c|c|c|c|c|c|}
\hline & $\mathrm{U}^{11}$ & $\mathrm{U}^{22}$ & $\mathrm{U}^{33}$ & $\mathrm{U}^{23}$ & $\mathrm{U}^{13}$ & $\mathrm{U}^{12}$ \\
\hline $\operatorname{Ir}(1)$ & $28(1)$ & $32(1)$ & $32(1)$ & $-4(1)$ & $9(1)$ & $-3(1)$ \\
\hline $\mathrm{Cl}(1)$ & $197(5)$ & $327(7)$ & $216(5)$ & $-63(5)$ & $-9(4)$ & $147(5)$ \\
\hline $\mathrm{Cl}(2)$ & $199(5)$ & $165(4)$ & $274(6)$ & $-73(4)$ & $-60(4)$ & $5(4)$ \\
\hline $\mathrm{Cl}(3)$ & $147(3)$ & $301(5)$ & $128(3)$ & $75(3)$ & $-26(2)$ & $-123(4)$ \\
\hline $\mathrm{S}(1)$ & $66(1)$ & $51(1)$ & $67(1)$ & $-5(1)$ & $27(1)$ & $-5(1)$ \\
\hline $\mathrm{F}(1)$ & $129(5)$ & $140(5)$ & 76(4) & $22(3)$ & $-27(3)$ & $27(4)$ \\
\hline $\mathrm{F}(2)$ & $96(4)$ & $116(4)$ & $96(4)$ & $8(3)$ & $53(3)$ & $-8(3)$ \\
\hline $\mathrm{F}(3)$ & $168(5)$ & $73(3)$ & $70(3)$ & $-15(2)$ & $47(3)$ & 4(3) \\
\hline $\mathrm{O}(1)$ & $90(4)$ & $49(3)$ & 92(4) & $-10(3)$ & $31(3)$ & $-3(3)$ \\
\hline $\mathrm{O}(2)$ & $94(4)$ & $96(4)$ & $68(4)$ & $8(3)$ & $9(3)$ & $32(3)$ \\
\hline $\mathrm{O}(3)$ & $82(4)$ & $77(4)$ & $99(5)$ & $-10(3)$ & $46(3)$ & $-15(3)$ \\
\hline $\mathrm{N}(1)$ & $45(3)$ & $35(2)$ & $39(3)$ & $-2(2)$ & $14(2)$ & $6(2)$ \\
\hline $\mathrm{N}(2)$ & $26(2)$ & $38(2)$ & $28(2)$ & $-3(2)$ & $3(2)$ & $0(2)$ \\
\hline $\mathrm{N}(3)$ & $45(3)$ & $44(3)$ & $35(3)$ & $-7(2)$ & $5(2)$ & $-5(2)$ \\
\hline N(4) & $42(3)$ & $42(2)$ & $39(3)$ & $1(2)$ & $11(2)$ & $-8(2)$ \\
\hline$C(1)$ & $67(4)$ & $40(3)$ & $66(4)$ & $-12(3)$ & $19(3)$ & $3(3)$ \\
\hline$C(2)$ & $95(7)$ & $64(5)$ & $95(6)$ & $-16(4)$ & $33(5)$ & $29(5)$ \\
\hline$C(3)$ & $78(6)$ & $65(5)$ & $115(7)$ & $-4(5)$ & $34(5)$ & $40(5)$ \\
\hline $\mathrm{C}(4)$ & $53(4)$ & $77(5)$ & $83(6)$ & $-2(4)$ & $17(4)$ & $25(4)$ \\
\hline
\end{tabular}




\begin{tabular}{|c|c|c|c|c|c|c|}
\hline$C(5)$ & $36(3)$ & $47(3)$ & $51(4)$ & $3(3)$ & $16(2)$ & $6(3)$ \\
\hline$C(6)$ & $29(2)$ & $44(3)$ & $43(3)$ & $6(2)$ & $11(2)$ & $-1(2)$ \\
\hline$C(7)$ & $31(3)$ & $65(4)$ & $51(4)$ & $13(3)$ & $2(2)$ & $0(3)$ \\
\hline$C(8)$ & $40(3)$ & $66(4)$ & $48(4)$ & $8(3)$ & $-9(3)$ & $-14(3)$ \\
\hline $\mathrm{C}(9)$ & $51(3)$ & $56(4)$ & $39(3)$ & $-4(3)$ & $-7(3)$ & $-8(3)$ \\
\hline$C(10)$ & $40(3)$ & $41(3)$ & 41(3) & $-4(2)$ & $2(2)$ & $-3(2)$ \\
\hline $\mathrm{C}(11)$ & $33(3)$ & $38(3)$ & $36(3)$ & $3(2)$ & $11(2)$ & $0(2)$ \\
\hline$C(12)$ & $93(6)$ & $82(5)$ & $65(5)$ & $-34(4)$ & $-25(4)$ & $-3(5)$ \\
\hline$C(13)$ & $37(3)$ & $51(3)$ & $45(3)$ & $-2(3)$ & $13(2)$ & $-3(3)$ \\
\hline$C(14)$ & $35(3)$ & $63(4)$ & $64(4)$ & $-12(3)$ & $18(3)$ & $8(3)$ \\
\hline$C(15)$ & $55(4)$ & $60(4)$ & $66(5)$ & $2(4)$ & 11(3) & $19(3)$ \\
\hline$C(16)$ & $56(4)$ & $50(4)$ & $57(4)$ & $7(3)$ & $11(3)$ & $12(3)$ \\
\hline$C(17)$ & $39(3)$ & $39(3)$ & $34(3)$ & $3(2)$ & $0(2)$ & $5(2)$ \\
\hline $\mathrm{C}(18)$ & $41(3)$ & $43(3)$ & $33(3)$ & $3(2)$ & $6(2)$ & $-3(2)$ \\
\hline$C(19)$ & $79(5)$ & $46(3)$ & $46(4)$ & $8(3)$ & $19(3)$ & $0(3)$ \\
\hline $\mathrm{C}(20)$ & $81(5)$ & $67(4)$ & $54(4)$ & 11(3) & $31(4)$ & $-10(4)$ \\
\hline$C(21)$ & $64(4)$ & $66(4)$ & $51(4)$ & $0(3)$ & $33(3)$ & $-11(3)$ \\
\hline$C(22)$ & $49(3)$ & $48(3)$ & $42(3)$ & $-9(3)$ & $19(3)$ & $-7(3)$ \\
\hline$C(23)$ & $30(2)$ & $40(3)$ & $35(3)$ & $-4(2)$ & $10(2)$ & $-7(2)$ \\
\hline$C(24)$ & $103(7)$ & $111(7)$ & $74(6)$ & $-10(5)$ & $63(5)$ & $-10(5)$ \\
\hline$C(25)$ & $42(3)$ & $32(3)$ & $39(3)$ & $-6(2)$ & $14(2)$ & $-8(2)$ \\
\hline$C(26)$ & $63(4)$ & $58(4)$ & $37(3)$ & $-8(3)$ & $-4(3)$ & $2(3)$ \\
\hline$C(27)$ & $68(5)$ & $89(6)$ & $61(5)$ & $-18(4)$ & $-9(4)$ & $-25(4)$ \\
\hline C(28) & $99(6)$ & $105(6)$ & $42(4)$ & $-20(4)$ & $16(4)$ & $-6(5)$ \\
\hline C(29) & $107(7)$ & $77(5)$ & $70(6)$ & $-4(4)$ & $-20(5)$ & $14(5)$ \\
\hline$C(30)$ & $29(3)$ & $35(3)$ & $47(3)$ & $-7(2)$ & $11(2)$ & $-3(2)$ \\
\hline $\mathrm{C}(31)$ & $75(5)$ & $58(4)$ & $33(3)$ & $9(3)$ & $14(3)$ & $0(3)$ \\
\hline$C(32)$ & $105(7)$ & $128(8)$ & $77(6)$ & $38(6)$ & $5(5)$ & $38(7)$ \\
\hline$C(33)$ & $151(9)$ & $86(6)$ & $43(4)$ & $-17(4)$ & $18(5)$ & $-12(6)$ \\
\hline$C(34)$ & $100(6)$ & $87(6)$ & $63(5)$ & $15(4)$ & $41(4)$ & $-22(5)$ \\
\hline$C(35)$ & $91(7)$ & $131(8)$ & $67(6)$ & $-7(5)$ & $12(5)$ & $-9(6)$ \\
\hline$C(36)$ & $62(4)$ & $63(4)$ & $56(4)$ & $8(3)$ & $5(3)$ & $0(4)$ \\
\hline
\end{tabular}


Table S 13. Hydrogen coordinates ( $\mathrm{x}$ 104) and isotropic displacement parameters ( $\AA 2 \mathrm{x} 103)$ for $[($ tpy $) 2 \operatorname{Ir}(\mathrm{CN}-\mathrm{t}-\mathrm{Bu}) 2](\mathrm{CF} 3 \mathrm{SO} 3) \cdot \mathrm{CHCl} 3$.

\begin{tabular}{|c|c|c|c|c|}
\hline & $\mathrm{x}$ & $\mathrm{y}$ & $\mathrm{z}$ & $\mathrm{U}(\mathrm{eq})$ \\
\hline $\mathrm{H}(1)$ & 4845 & 3774 & 8192 & 68 \\
\hline $\mathrm{H}(2)$ & 3536 & 4846 & 8249 & 99 \\
\hline $\mathrm{H}(3)$ & 2025 & 4561 & 7655 & 101 \\
\hline $\mathrm{H}(4)$ & 1852 & 3198 & 7005 & 85 \\
\hline $\mathrm{H}(7)$ & 1900 & 1952 & 6341 & 59 \\
\hline $\mathrm{H}(8)$ & 2063 & 623 & 5704 & 64 \\
\hline $\mathrm{H}(10)$ & 4829 & 74 & 6560 & 49 \\
\hline $\mathrm{H}(12 \mathrm{~A})$ & 3216 & -1206 & 5733 & 125 \\
\hline $\mathrm{H}(12 \mathrm{~B})$ & 4290 & -789 & 5657 & 125 \\
\hline $\mathrm{H}(12 \mathrm{C})$ & 3330 & -397 & 5231 & 125 \\
\hline $\mathrm{H}(13)$ & 6957 & 947 & 6943 & 53 \\
\hline $\mathrm{H}(14)$ & 7809 & -557 & 6903 & 63 \\
\hline $\mathrm{H}(15)$ & 7397 & -1930 & 7458 & 72 \\
\hline $\mathrm{H}(16)$ & 6198 & -1755 & 8062 & 65 \\
\hline $\mathrm{H}(19)$ & 5161 & -1386 & 8686 & 67 \\
\hline $\mathrm{H}(20)$ & 4080 & -902 & 9320 & 79 \\
\hline $\mathrm{H}(22)$ & 3735 & 1817 & 8520 & 54 \\
\hline $\mathrm{H}(24 \mathrm{~A})$ & 2690 & 282 & 9489 & 136 \\
\hline $\mathrm{H}(24 \mathrm{~B})$ & 2739 & 1421 & 9264 & 136 \\
\hline $\mathrm{H}(24 \mathrm{C})$ & 3531 & 1026 & 9800 & 136 \\
\hline $\mathrm{H}(27 \mathrm{~A})$ & 8443 & 3174 & 9137 & 112 \\
\hline $\mathrm{H}(27 \mathrm{~B})$ & 8196 & 3429 & 9780 & 112 \\
\hline $\mathrm{H}(27 \mathrm{C})$ & 7624 & 4004 & 9218 & 112 \\
\hline $\mathrm{H}(28 \mathrm{~A})$ & 6024 & 3477 & 9507 & 122 \\
\hline $\mathrm{H}(28 \mathrm{~B})$ & 6548 & 2833 & 10055 & 122 \\
\hline $\mathrm{H}(28 \mathrm{C})$ & 5773 & 2305 & 9556 & 122 \\
\hline $\mathrm{H}(29 \mathrm{~A})$ & 7005 & 1026 & 9430 & 132 \\
\hline $\mathrm{H}(29 \mathrm{~B})$ & 7889 & 1532 & 9869 & 132 \\
\hline $\mathrm{H}(29 \mathrm{C})$ & 8015 & 1327 & 9198 & 132 \\
\hline $\mathrm{H}(32 \mathrm{~A})$ & 4354 & 3424 & 5712 & 156 \\
\hline
\end{tabular}




\begin{tabular}{lllll}
$\mathrm{H}(32 \mathrm{~B})$ & 4891 & 4017 & 5241 & 156 \\
$\mathrm{H}(32 \mathrm{C})$ & 4965 & 4432 & 5900 & 156 \\
$\mathrm{H}(33 \mathrm{~A})$ & 6425 & 1824 & 5635 & 139 \\
$\mathrm{H}(33 \mathrm{~B})$ & 5958 & 2443 & 5062 & 139 \\
$\mathrm{H}(33 \mathrm{C})$ & 5248 & 1895 & 5458 & 139 \\
$\mathrm{H}(34 \mathrm{~A})$ & 6834 & 4352 & 6121 & 120 \\
$\mathrm{H}(34 \mathrm{~B})$ & 6793 & 4111 & 5435 & 120 \\
$\mathrm{H}(34 \mathrm{C})$ & 7379 & 3373 & 5918 & 120 \\
$\mathrm{H}(35)$ & 247 & 1660 & 7544 & 116 \\
\hline
\end{tabular}

